\title{
Zur Berechnung dünnwandiger Stäbe mit variablem, offenem Querschnitt
}

\section{Working Paper}

\section{Author(s):}

Karamuk, Ergun

Publication date:

1968

Permanent link:

https://doi.org/10.3929/ethz-a-000747207

Rights / license:

In Copyright - Non-Commercial Use Permitted

Originally published in:

Bericht / Institut für Baustatik ETH Zürich 17 
Zur Berechnung dünnwandiger Stäbe mit variablem, offenem Querschnitt

Ergun Karamuk 


\title{
Zur Berechnung dünnwandiger Stäbe mit variablem, offenem Querschnitt
}

\author{
von \\ Dr. sc. techn. Ergun Karamuk \\ Institut für Baustatik \\ Eidgenössische Technische Hochschule Zürich
}

Zürich

September 1968 
Leer - Vide - Empty 


\section{Leer - Vide - Empty}




$$
\text { Leer - Vide - Empty }
$$


TORWORT

Bricken at Plettenbelkenquerechnitt werden houte aohr oft al. dinnwand1ge Stabe ait offonen Quersehnitt berechnot. Die Toralonsbelastung soloher Tragrer'e bereltet bel konstanten Querschnitt keine Schwierigkeiten. Ist Jedooh die Hrhe verinderlich, so konnen in stab je nach Iagerang anoh B1egemomente entetehen, die von der roralonbbelantung herrthren.

Die rorliegende theoretieche Untersuohung dieaes Problene warde von Herm Brgun Karanuk als Diesertation (Reforent Prof. Dr. B. Thilrifmann, Korreferent Prof. Dr. P. Dubae) ansgearbeltet. Anhand oines Kodellos wird olne einfache Berechnungamethode sur Ibsung des Toreioneprobleme dinnwandger stube nit rariablen, offonom Querwohnitt entwickelt. Der wohtige Ififluse der Lagerang auf das Verhalton soloher stube wird anscheulich dikutiert. Die srbeit direfte besonders bel der prakt1sohen Berechnung von Brickon mit offenen, variablen Quersobnitt Inrondung findon.

Bidgonosalwohe Teohntsohe Hochschule 


$$
\text { Leer - Vide - Empty }
$$


1. EINLEITUNG

Seite

2. VORAUSSETZUNGEN 12

3. DIFFERENTIALGLEICHUNGEN 14

3.1 Herleitung der Differentialgleichungen 14

3.2 Einfluss der Lagerung 17

$\begin{array}{ll}3.3 \text { Lösungsmethoden } & 19\end{array}$

3.4 Modell 20

4. VERNACHLAESSIGUNG DER ST. VENANTSCHEN TORSION 27

4.1 Verhalten unter Torsion 27

4.2 Beliebige Lagerung 31

4.3 Durchlaufender Stab 41

5. VERGLEICH MIT DER FALTWERKTHEORIE 42

5.1 Berechnung als Faltwerk 42

5.2 Beispiele 48

6. BERUECKSICHTIGUNG DER ST. VENANTSCHEN TORSION

6.1 Praktische Berechnung 54

6.2 Beispiele 56

7. ALLGEMEINE VERAENDERLICHKEIT DES QUERSCHNITTS 58

8. ZUSAMMENFASSUNG 62

ANHANG 63

1. Einfluss der veränderlichen Scheibenhöhe 63

2. Konzentrierte Schubkräfte bei Querschnittssprüngen 65

3. Berechnung des Beispiels in Bild $21 \quad 67$

BEZEICHNUNGEN

LITERATUR - VERZEICHNIS 


$$
\text { Leer - Vide - Empty }
$$




\section{EINLEITUNG}

Das Verhalten dünnwandiger Stäbe mit konstantem, offenem Querschnitt unter Torsionsbelastung ist in den letzten vier Jahrzehnten intensiv erforscht worden. Von den zahlreichen Publikationen seien hier nur die grundlegende Arbeit von W.S. WLASSOW [1] und die einfache Darstellung von C.F. KOLLBRUNNER / K. BASLER [2] genannt, in denen auch die wichtigsten Literaturangaben zu finden sind.

Die Torsion des duinnwandigen Stabes mit beliebig variablem, offenem Querschnitt hingegen wurde bisher wenig behandelt. Z. CYWINSKI [3] untersucht einen zylindrischen Stab mit ofienem, dünwandigem Querschnitt, dessen Querschnittsränder beliebige Raumkurven sind. Er stellt die Differentialgleichungen des Torsionsproblems auf und löst sie für den Fall des einfachsymmetrischen Querschnittes mit Hilfe der Differenzenmethode. Z.P. BAZANT [4] zeigt die Herleitung der Differentialgleichungen fuir Stäbe mit einfachsymmetrischen Querschnitten unter beliebiger äusserer Belastung. Einen in einer Ebene gekrimmten Stab mit stückweise konstantem, einfachsymmetrischem Querschnitt berechnet G. BECKER [5] mit Hilfe des Reduktionsverfahrens. Die Querschnittsveränderlichkeit wird hier bei der Formulierung der Uebergangsbedingungen bericksichtigt. Auch S. LONKAR [6] schlägt einen ähnlichen Weg vor, indem er von den einfacheren Differentialgleichungen des geraden Trägers ausgehend beliebige Querschnittsformen behandelt und den gekrümmten Stab durch ein Polygon approximiert. Der Spezialfall des I-Trägers mit veränderlicher Steghöhe wird von G.C. LEE und B.A. SZABO [7] untersucht. 
Im Gegensatz zum prismatischen Stab treten beim dünnwandigen Träger mit variablem, offenem Querschnitt unter äusserer Torsionsbelastung im allgemeinen Fall auch Biegemomente auf, so dass Biegung und Verdrehung sich gegenseitig beeinflussen. Das Torsionsproblem wird nicht mehr von einer Differentialgleichung, sondern von einem System von vier Differentialgleichungen beschrieben.

Wird ein durch äussere Lasten belasteter, dünnwandiger Stab auf ein beliebiges, rechtwinkliges Koordinatensystem bezogen (Bild 1), so können die Spannungen und somit die Schnittkräfte im Querschnitt $z=$ konst. durch die vier Funktionen $\zeta(z), \xi(z), \eta(z)$ und $\varphi(z)$ dargestellt werden. Dabei bedeutet $\zeta(z)$ die Verschiebung des Querschnittes in $z$-Richtung, $\xi(z)$ und $\eta(z)$ die Verschiebungen des Pols $A$ in $x$ - und $y$-Richtung und $\varphi(z)$ die Verdrehung des Querschnittes um diesen Pol, welcher der Durchstosspunkt der z-Achse mit der Querschnittsebene ist. Diese vier unbekannten Funktionen können aus einem System von vier linearen Differentialgleichungen vierter Ordnung, mit variablen Koeffizienten gewonnen werden.

Bei prismatischen Stäben und solchen mit veränderlichen aber doppelsymmetrischen Querschnitten (Bild 2) ist es immer möglich, durch die Wahl eines speziellen Bezugssystems diese Differentialgleichungen zu entkoppeln, so dass vier voneinander unabhängige Differentialgleichungen entstehen. Eine dieser Gleichungen beschreibt die Torsion des Stabes, die übrigen beschreiben die

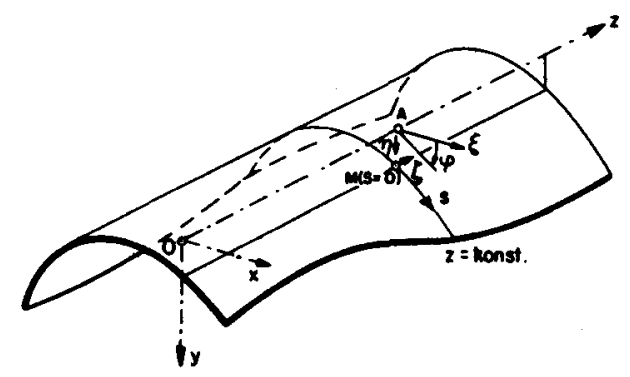

Bild 1 Koordinatensystem und positiv definierte Deformationen des Querschnitts 
Längenänderung und die Durchbiegungen in $x$ - und $y$-Richtung. Hat der Stab variable, einfachsymmetrische Querschnitte, so kann das System von vier Differentialgleichungen auf zwei unabhängige Systeme von je zwei Differentialgleichungen reduziert werden.

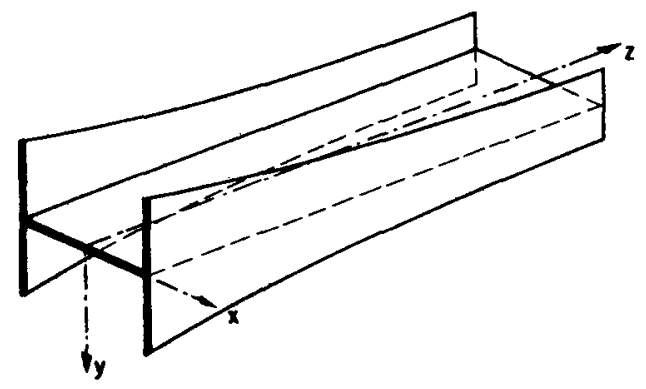

Bild 2 Stab mit veränderlichem Querschnitt und gerader Schubmittelpunktsachse (doppelsymmetrischer Querschnitt)

Die Entkoppelung des Differentialgleichungssystems ist bei Stäben mit beliebig veränderlichen Querschnitten im allgemeinen nicht möglich. Diese Gleichungen werden normalerweise numerisch gelöst. Erlaubt jedoch die Lagerung des durch äussere Torsionsmomente belasteten Stabes zwängungsfreie Verformungen in Richtung der Koordinatenachsen, so gelingt es, das System auf eine einzige Differentialgleichung zurückzuführen, die gleich aufgebaut ist wie diejenige des prismatischen Stabes. Ihre Koeffizienten sind aber nicht mehr konstant, sondern Funktionen der Variablen z. Im allgemeinen kann diese Differentialgleichung nicht geschlossen gelöst werden und wird daher numerisch behandelt.

Das Ziel der vorliegenden Arbeit ist, im Rahmen der technischen Biege- und Torsionslehre einfache Berechnungsmethoden zur Lösung des Torsionsproblems dünnwandiger stäbe mit variablem, offenem Querschnitt zu entwickeln und den wichtigen Einfluss der Lagerung auf das Verhalten solcher Stäbe zu zeigen. Dabei wird der zwängungsfrei gelagerte Träger als Grundsystem gewählt, der unter Vernachlässigung der St. Venantschen Torsionssteifigkeit auch nach der Faltwerktheorie gerechnet wird. Fïr die numerische Iösung der Differentialgleichung wird ein Modell zu Hilfe genommen. 


\section{VORAUSSETZUNGEN}

Für die Berechnung des dünwandigen Stabes wird folgendes vorausgesetzt:

1. Die grössten Querschnittsabmessungen sind klein im Vergleich zur Stablänge.

2. Das Material ist linear elastisch. (Hookesches Gesetz)

3. Die Deformationen sind klein.

4. Die Querschnittsform bleibt erhalten.

5. Die Schubverformung aus den Wölbschubspannungen wird vernachlässigt.

6. Die Mittelflächen der Wandelemente des dünwandigen Stabes sind parallel zur Stablängsrichtung, d.h. der Stab wird aus einem prismatischen Körper herausgeschnitten.

7. Die Veränderlichkeit des Querschnittes in Stablängsrichtung ist schwach, so dass die Spannungen und Dehnungen der Faserm senkrecht zur Querschnittsebene angenommen werden.

Neben den bekannten Voraussetzungen der Stabstatik (eins bis drei) basiert die Theorie der dünnwandigen Stäbe mit offenem Querschnitt auf den Annahmen vier und fünf. Hier kommt als sechste Voraussetzung die besondere Veränderlichkeit des Querschnittes hinzu. Bild 1 und Bild 2 sind Beispiele für solche Stäbe. Sind die Mittelflächen der Wandelemente nicht parallel zur Stablängsrichtung, wie das beim I-Träger mit variabler Steghöhe der Fall ist, dann ist die Voraussetzung sieben nicht mehr erfillt. In Kapitel 7 wird diese Einschränkung der Querschnittsveränderlichkeit näher behandelt. Die Gültigkeit der Voraussetzung sieben wird im Anhang untersucht.

Für die praktische Durchführung der Berechnung wird der Stab mit variablem Querschnitt durch einen solchen ersetzt, der aus prismatischen Teilstücken zusammengesetzt ist. Diese Approximation, die auch bei [5] und [6] gemacht wird, ist nur dann zulässig, wenn die beiden letzten Voraussetzungen erfült sind, was in den meisten praktischen Fällen zutrifft. 


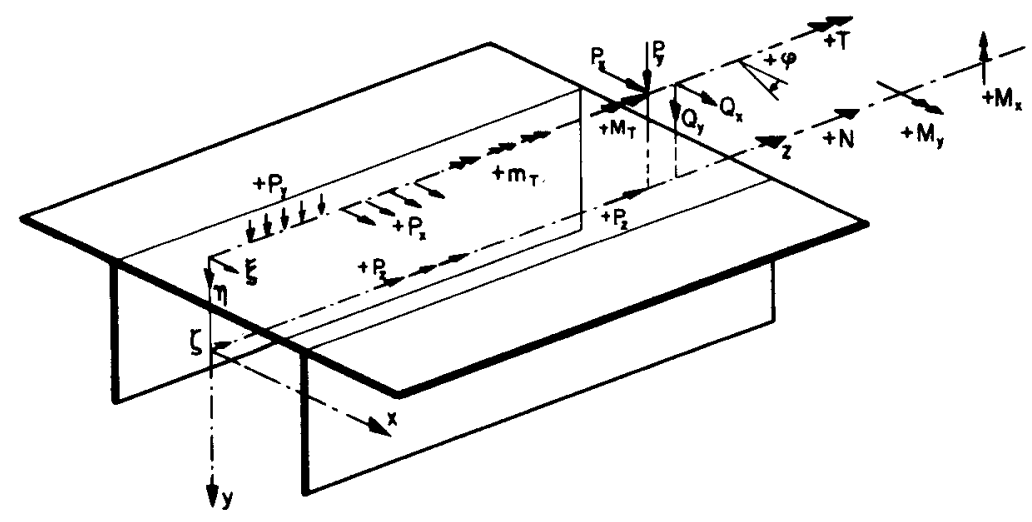

Bild 3 Positiv definierte Belastungen, Schnittkräfte und Deformationen

Das der Berechnung zugrunde gelegte Koordinatensystem ist in Bild 3 dargestellt, aus der auch die positiv definierten Belastungen, Schnittkräfte und Deformationen hervorgehen.*)

Die Biegemomente $M_{x}, M_{y}$, die Normalkraft $N$ und die Längsverschiebung $\zeta$ werden auf die Schwerachse, die Querkräfte $Q_{x}, Q_{y}$, das Torsionsmoment $T$ und die Verschiebungen $\xi$ und $\eta$ parallel zu den Koordinatenachsen $\mathrm{x}$ und $\mathrm{y}$ auf die Schubmittelpunktsachse bezogen.

*) Die Biegemomente werden folgendermassen definiert:

$$
\mathrm{M}_{\mathrm{X}}=\int_{F} \sigma \mathrm{x} d \mathrm{~F} \quad, \quad \mathrm{M}_{\mathrm{y}}=\int_{F} \sigma \mathrm{y} d \mathrm{~F}
$$


3. DIFFERENTIALGLEICHUNGEN

\subsection{Herleitung der Differentialgleichungen}

Im folgenden wird vollständigkeitshalber die Herleitung der bekannten Differentialgleichungen des dïnnwandigen Stabes mit offenem Profil wiedergegeben. Diese können entweder aus dem Gleichgewicht am Stabelement (z.B. in [1]) oder nach dem Minimumprinzip der potentiellen Energie (z.B. in [3] und [8] ) gewonnen werden. Hier wird die einfachere, zweite Methode gewählt.

Die potentielle Energie (Potential $\pi$ ) ist die Summe der aufgespeicherten Formänderungsarbeit (inneres Potential $\pi_{i}$ ) und der negativen Arbeit der Lasten (äusseres Potential $\pi_{a}$ ).

$$
\pi=\pi_{i}+\pi_{a}
$$

Das innere Potential $\pi_{i}$ des dinnwandigen Stabes ist:

$$
\Pi_{i}=\frac{I}{2 E} \int_{V} \sigma^{2} d V+\frac{1}{2 G} \int_{V} \tau^{2} d V
$$

Mit der verteilten Torsionsbelastung $\mathrm{m}(\mathrm{z})$ lautet das äussere Potential $\Pi_{a}$ :

$$
\pi_{a}=-\int_{0}^{l} m(z) \varphi d z
$$

Gemäss Voraussetzung fünf wird der Verformungseinfluss der Wölbschubspannungen vernachlässigt, so dass nur die St. Venantschen Schubspannungen $\tau_{s}$ einen Beitrag zum inneren Potential leisten. Die Formänderungsarbeit dieser Schubspannungen ist gleich der Arbeit des St. Venantschen Torsionsmoments $T_{S}$, welches das resultierende Moment der Schubkräfte $\tau_{s} \mathrm{dF}$ im Querschnitt $z$ = konst. ist. Deshalb kann der zweite Summand von Gl. (2) folgendermassen geschrieben werden: 


$$
\pi_{i}^{s}=\frac{1}{2} \int_{0}^{\ell} T_{s} \varphi^{\prime} \mathrm{dz}
$$

Mit $\mathrm{T}_{\mathbf{S}}=\mathrm{GK} \boldsymbol{\varphi}^{\prime}$ wird

$$
\Pi_{i}^{s}=\frac{1}{2} \int_{0}^{\ell} G K \varphi^{\prime 2} \mathrm{dz}
$$

Die Normalspannungen in einem beliebigen Punkt des Stabquerschnittes $\mathbf{z}=$ konst. (Bild 1) werden mit der Beziehung

$$
\sigma(z, s)=E\left[\zeta^{\prime}(z)-\xi^{\prime \prime}(z) x(s)-\eta^{\prime \prime}(z) y(s)-\varphi^{\prime \prime}(z) \omega(s)\right]
$$

ausgedrückt. Wird in GI. (2) für $\sigma$ die Beziehung (6) eingesetzt und der zweite Summand durch Gl. (5) ersetzt, so entsteht nach Gl. (1) mit den Abkürzungen

$$
\begin{aligned}
F=\int_{F} d F, \quad I_{x x} & =\int_{F} x^{2} d F, & I_{y y}=\int_{F} y^{2} d F, & I_{\omega \omega}=\int_{F} \omega^{2} d F, \\
I_{x y} & =\int_{F} x y d F, & I_{\omega x}=\int_{F} \omega x d F, & I_{\omega y}=\int_{F} \omega y d F \\
S_{x} & =\int_{F} x d F, & S_{y}=\int_{F} y d F, & S_{\omega}=\int_{F} \omega d F
\end{aligned}
$$

folgender Ausdruck für das Potential :

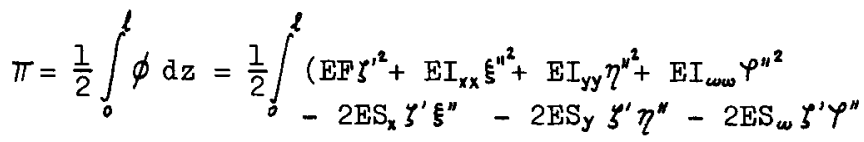

$$
\begin{aligned}
& +2 \mathrm{EI} I_{x y} \xi^{\prime \prime} \eta^{\prime \prime}+2 \mathrm{EI} I_{w x} \xi^{\prime \prime} \varphi^{\prime \prime}+2 \mathrm{EI} I_{w y} \eta^{\prime \prime} \varphi^{\prime \prime} \\
& \left.+\mathrm{GK} \varphi^{\prime 2}-2 \mathrm{~m}(\mathrm{z}) \varphi\right) d z
\end{aligned}
$$

Das Potential des Stabes muss ein Minimum sein. Das ist der Fall, wenn die vier Eulerschen Differentialgleichungen (8) erfüllt werden. 


$$
\begin{aligned}
& \frac{\partial \phi}{\partial \zeta}-\frac{d}{d z}\left(\frac{\partial \phi}{\partial \zeta^{\prime}}\right)+\frac{d^{2}}{d z^{2}}\left(\frac{\partial \phi}{\partial \zeta^{\prime \prime}}\right)=0 \\
& \frac{\partial \phi}{\partial \xi}-\frac{d}{d z}\left(\frac{\partial \phi}{\partial \xi^{\prime}}\right)+\frac{d^{2}}{d z^{2}}\left(\frac{\partial \phi}{\partial \xi^{\prime \prime}}\right)=0 \\
& \frac{\partial \phi}{\partial \eta}-\frac{d}{d z}\left(\frac{\partial \phi}{\partial \eta^{\prime}}\right)+\frac{d^{2}}{d z^{2}}\left(\frac{\partial \phi}{\partial \eta^{4}}\right)=0 \\
& \frac{\partial \phi}{\partial \varphi}-\frac{d}{d z}\left(\frac{\partial \phi}{\partial \varphi^{\prime \prime}}\right)+\frac{d^{2}}{d z^{2}}\left(\frac{\partial \phi}{\partial \varphi^{\prime \prime}}\right)=0
\end{aligned}
$$

Die Differentialgleichungen des Torsionsproblems lauten:

$$
\begin{aligned}
&\left(\mathrm{EF} \zeta^{\prime}\right)^{\prime}-\left(\mathrm{ES}_{x} \xi^{\prime \prime}\right)^{\prime}-\left(\mathrm{ES}_{y} \eta^{\prime \prime}\right)^{\prime}-\left(\mathrm{ES}_{\omega} \varphi^{\prime \prime}\right)^{\prime}=0 \\
&-\left(\mathrm{ES}_{x} \zeta^{\prime}\right)^{\prime \prime}+\left(\mathrm{EI}_{x x} \xi^{\prime \prime}\right)^{\prime \prime}+\left(\mathrm{EI}_{x y} \eta^{\prime \prime}\right)^{\prime \prime}+\left(\mathrm{EI}_{\omega x} \varphi^{\prime \prime}\right)^{\prime \prime}=0 \\
&-\left(\operatorname{ES}_{y} \zeta^{\prime}\right)^{\prime \prime}+\left(\mathrm{EI}_{x y} \xi^{\prime \prime}\right)^{\prime \prime}+\left(\mathrm{EI}_{y y} \eta^{\prime \prime}\right)^{\prime \prime}+\left(\mathrm{EI}_{\omega y} \varphi^{\prime \prime}\right)^{\prime \prime}=0 \\
&-\left(\mathrm{ES}_{\omega} \zeta^{\prime}\right)^{\prime \prime}+\left(\mathrm{EI}_{\omega x} \xi^{\prime \prime}\right)^{\prime \prime}+\left(\mathrm{EI}_{\omega y} \eta^{\prime \prime}\right)^{\prime \prime}+\left(\mathrm{EI}_{w \omega} \varphi^{\prime \prime}\right)^{\prime \prime}-\left(\mathrm{GK} \varphi^{\prime}\right)^{\prime}=\mathrm{m}(z)
\end{aligned}
$$

Bei allgemeiner Belastung kommen auf der rechten Seite des Gleichungssystems (9) statt Null die äusseren Lasten $p_{z}(z), p_{X}(z)$ und $\mathrm{p}_{\mathrm{y}}(\mathrm{z})$ vor. Bei prismatischen stäben und solchen mit doppelsymmetrischen Querschnitten (Bild 2) kann durch die Wahl eines speziellen Bezugssystems $S_{x}=S_{y}=S_{\omega}=I_{x y}=I_{\omega x}=I_{\omega y}=0$ gesetzt werden. In diesem Fall zerfällt das Gleichungssystem (9) in vier unabhängige Gleichungen. Die vierte Gleichung ist die bekannte Differentialgleichung der Torsion, jedoch mit variablen Koeffizienten. Bei Stäben mit einfachsymmtrischen Querschnitten kann dieses System auf zwei Teilsysteme mit je zwei Unbekannten reduziert werden. Mit der $y$-Achse als Symmetrieachse wird $S_{x}=S_{\omega}=I_{x y}=I_{w y}=0$, und das Gleichungssystem, das das Torsionsproblem beschreibt, nimmt folgende Form an:

$$
\begin{aligned}
& \left(\mathrm{EI}_{x x} \xi^{\prime \prime}\right)^{\prime \prime}+\left(\mathrm{EI} \mathrm{I}_{\omega x} \varphi^{\prime \prime}\right)^{\prime \prime}=0 \\
& \left(E I_{\omega x} \xi^{\prime \prime}\right)^{\prime \prime}+\left(E I_{\omega \nu} \varphi^{\prime \prime \prime}\right)^{\prime \prime}-\left(G K \varphi^{\prime}\right)^{\prime}=0
\end{aligned}
$$

In [3] wurde dieses Gleichungssystem für ein Beispiel numerisch und für eine einfache Veränderlichkeit des Querschnittes und für $\mathrm{GK}=0$ exakt gelöst. 


\subsection{Einfluss der Lagerung}

Die Normalkraft $\mathrm{N}$ sowie die Biegemomente $\mathrm{M}_{\mathrm{x}}$ und $\mathrm{M}_{\mathrm{y}}$ im Querschnitt $z$ = konst. des in Bild 1 dargestellten Stabes nehmen folgende Formen an, wenn für $\sigma$ der Ausdruck (6) eingesetzt wird:

$$
\begin{aligned}
& N=\int_{F} \sigma d F=E F \zeta^{\prime}-E_{x} \xi^{\prime \prime}-E S_{y} \eta^{\prime \prime}-E^{\prime} S^{\prime \prime} \\
& \mathrm{M}_{\mathrm{x}}=\int_{F}^{F} \sigma \mathrm{xdF}=\mathrm{ES}_{x} \zeta^{\prime}-\mathrm{EI}_{x x} \xi^{\prime \prime}-\mathrm{EI}_{x y} \eta^{\prime \prime}-\mathrm{EI}_{\omega x} \varphi^{\prime \prime} \\
& \mathrm{M}_{y}=\int_{F} \sigma \mathrm{ydF}=\mathrm{ES} \mathrm{S}_{y} \zeta^{\prime}-\mathrm{EI}_{x y} \xi^{\prime \prime}-E I_{y y} \eta^{\prime \prime}-\mathrm{EI}_{w y} \varphi^{\prime \prime}
\end{aligned}
$$

Ein Vergleich mit dem Gleichungssystem (9) zeigt, dass die erste Ableitung von GI. (1la) mit der ersten, die zweiten Ableitungen von Gl. (IIb) und (IIc) mit der zweiten und dritten Gleichung des Systems ubereinstimmen.

Da die erste Ableitung der Normalkraft verschwindet, muss sie über die ganze Stablänge konstant oder gleich Null sein. Die Biegemomente sind höchstens lineare Funktionen von $z$, weil ihre zweiten Ableitungen verschwinden. Sie können aber auch konstant bleiben, und wenn die Lagerung an den Stabenden keine Biegemomente ubertragen kann, sind sie gleich Null.

Aus den Gleichungen (II) können die Unbekannten $\zeta^{\prime}$, $\xi$ " und $\eta^{\prime \prime}$ in Funktionen von $\varphi^{\prime \prime}$ dargestellt werden. Das Gleichungssystem lautet:

$$
\begin{aligned}
\mathrm{F} \xi^{\prime}-\mathrm{S}_{x} \xi^{\prime \prime}-\mathrm{S}_{y} \eta^{\prime \prime} & =\mathrm{S}_{\boldsymbol{\omega}} \varphi^{\prime \prime}+\mathrm{N} / \mathrm{E} \\
-\mathrm{S}_{x} \xi^{\prime}+\mathrm{I}_{x x} \xi^{\prime \prime}+I_{x y} \eta^{\prime \prime} & =-I_{\omega x} \varphi^{\prime \prime}-\mathrm{M}_{x} / \mathrm{E} \\
-S_{y} \xi^{\prime}+I_{x y} \xi^{\prime \prime}+I_{y y} \eta^{\prime \prime} & =-I_{\omega y} \varphi^{\prime \prime}-\mathrm{M}_{y} / \mathrm{E}
\end{aligned}
$$

Mit den Koeffizienten $a_{i k}$ der Kehrmatrix können die Unbekannten folgendermassen geschrieben werden:

$$
\begin{aligned}
& \zeta^{\prime}=a_{11}\left(S_{\omega} \varphi^{\prime \prime}+N / E\right)+a_{12}\left(-I_{\omega x} \varphi^{\prime \prime}-M_{x} / E\right)+a_{13}\left(-I_{\omega y} \varphi^{\prime \prime}-M_{y} / E\right) \\
& \xi^{\prime \prime}=a_{21}\left(S_{\omega} \varphi^{\prime \prime}+N / E\right)+a_{22}\left(-I_{\omega x} \varphi^{\prime \prime}-M_{x} / E\right)+a_{23}\left(-I_{\omega y} \varphi^{\prime \prime}-M_{y} / E\right) \\
& \eta^{\prime \prime}=a_{31}\left(S_{\omega} \varphi^{\prime \prime}+N / E\right)+a_{32}\left(-I_{\omega x} \varphi^{\prime \prime}-M_{x} / E\right)+a_{33}\left(-I_{\omega y} \varphi^{\prime \prime}-M_{y} / E\right)
\end{aligned}
$$


Eingesetzt in die letzte Gleichung des Differentialgleichungssystems entsteht daraus:

$$
\begin{aligned}
& {\left[-S_{\omega}\left(a_{11} N-a_{12} M_{x}-a_{13} M_{y}\right)-E S_{\omega} \varphi^{\prime \prime}\left(a_{11} S_{\omega}-a_{12} I_{\omega x}-a_{43} I_{\omega y}\right)+\right.} \\
& +I_{w x}\left(a_{21} N-a_{22} M_{x}-a_{23} M_{y}\right)+E I_{\omega x} \varphi^{\prime \prime}\left(a_{21} S_{\omega}-a_{22} I_{w x}-a_{23} I_{\omega y}\right)+ \\
& +I_{w y}\left(a_{31} N-a_{32} \mathbb{M}_{x}-a_{33} \mathbb{M}_{y}\right)+E I_{w y} P^{\prime \prime}\left(a_{31} S_{w}-a_{32} I_{w x}-a_{33} I_{w y}\right)+ \\
& \left.+E I_{w w} \varphi^{\prime \prime}\right]^{\prime \prime}-\left(G K \varphi^{\prime}\right)^{\prime}=m(z)
\end{aligned}
$$

Ist die Lagerung des Stabes so, dass unter Torsionsbelastung weder Normalkräfte noch Biegemomente auftreten, d.h. wenn es sich um einen Kragträger oder einen Stab mit Gabellagerung an beiden Enden handelt, dann verschwinden die klammerausdrücke mit $N, M_{X}$ und $M_{y}$ und die Gleichung (14) nimmt folgende Form an:

$\left\{\left[S_{\omega}\left(-a_{11} S_{\omega}+a_{12} I_{\omega x}+a_{13} I_{\omega y}\right)+I_{\omega x}\left(a_{21} S_{\omega}-a_{22} I_{\omega x}-a_{23} I_{\omega y}\right)+\right.\right.$
$\left.\left.+I\left(a_{31} S_{\omega}-a_{32} I_{\omega x}-a_{33} I_{\omega y}\right)+I_{\omega \omega}\right] E \varphi^{\prime \prime}\right\}^{\prime \prime}-\left(G K \varphi^{\prime}\right)^{\prime}=m(z)$

oder mit den Abkürzungen $\alpha, \alpha_{y},-\alpha_{x}$ für die Ausdrücke in den runden Klammern:

$$
\left[\left(\alpha S_{\omega}+\alpha_{y} I_{\omega x}-\alpha_{x} I_{\omega y}+I_{\omega w}\right) E \varphi^{\prime \prime}\right]^{\prime \prime}-\left(G K \varphi^{\prime}\right)^{\prime}=m(z)
$$

Wie es in [1] gezeigt wird, ist der Ausdruck in der runden Klammer von Gl. (16) nichts anderes als die Wolbkonstante, d.h. das sektorielle Trägheitsmoment $I_{\omega \omega, \mathbf{D}}$ bezüglich des Schubmittelpunktes (Hauptsektorträgheitsmoment), wobei $\alpha_{x}$ und $\alpha_{y}$ die Koordinaten des Schubmittelpunktes $D$ sind und $\alpha=\alpha_{x} y\left(s_{0}\right)-\alpha_{y} x\left(s_{0}\right)$ ist. $x\left(s_{0}\right)$ und $\mathrm{y}\left(s_{0}\right)$ sind die Koordinaten des Sektoranfangspunktes $M$, für den $\omega_{A}=0$ ist (Bild 1 ). Zwischen den Querschnittswerten bezogen auf ein beliebiges Koordinatensystem $x, y$ und auf den Pol $A$ bestehen folgende Zusammenhänge: 


$$
\begin{aligned}
& S_{\omega}=-S_{x} \alpha_{y}+S_{y} \alpha_{x}-F \alpha \\
& I_{w x}=-I_{x x} \alpha_{y}+I_{x y} \alpha_{x}-S_{x} \alpha \\
& I_{w y}=-I_{x y} \alpha_{y}+I_{y y} \alpha_{x}-S_{y} \alpha
\end{aligned}
$$

Mit der Determinante

$$
D=F I_{x x} I_{y y}-F I_{x y}^{2}-S_{x}^{2} I_{y y}-S_{y}^{2} I_{x x}+2 S_{x} S_{y} I_{x y}
$$

des Gleichungssystems (12) Lauten die Koeffizienten der Kehrmatrix:

$$
\begin{array}{ll}
a_{11}=\left(I_{x x} I_{y y}-I_{x y}^{2}\right) / D, & a_{12}=a_{21}=\left(S_{x} I_{y y}-S_{y} I_{x y}\right) / D \\
a_{22}=\left(F I_{y y}-S_{y}^{2}\right) / D, & a_{13}=a_{31}=\left(S_{y} I_{x x}-S_{x} I_{x y}\right) / D \\
a_{33}=\left(F I_{x x}-S_{x}^{2}\right) / D, & a_{23}=a_{32}=\left(S_{x} S_{y}-F I_{x y}\right) / D
\end{array}
$$

Werden nun diese Koeffizienten $a_{i k}$ und die Querschnittswerte $s_{\omega}$, $I_{\omega x}$ und $I_{\omega y}$ aus GI. (17) in GI. (15) eingesetzt, so ergeben die Ausdrücke in den runden Klammern tatsächlich $\alpha, \alpha_{y}$ und $-\alpha_{x}$. Gleichung (16) kann daher einfacher geschrieben werden:

$$
\left(E I_{w \omega, D} \varphi^{\prime \prime}\right)^{\prime \prime}-\left(\mathrm{GK} \varphi^{\prime}\right)^{\prime}=\mathrm{m}(z)
$$

Es ist somit gezeigt, dass bei derjenigen Lagerung des dünwandigen Stabes mit variablem Querschnitt, bei der unter äusserer Torsionsbelastung keine Normalkräfte und Biegemomente entstehen können, das Torsionsproblem durch eine einzige Differentialgleichung beschrieben wird, die gleich aufgebaut ist wie die Torsionsdifferentialgleichung des prismatischen Stabes.

\subsection{Lösungsmethoden}

Die Differentialgleichung (18) bildet hier die Grundlage für die weitere Berechnung. Beliebig gelagerte Stäbe mit variablem Querschnitt werden auf Grundsysteme zurïckgeführt, für welche die 
Differentialgleichung (18) gültig ist, d.h. auf den Kragträger oder den Stab mit Gabellagerung an den Enden.

Bei prismatischen Stäben sind die Koeffizienten der Gleichung (18) konstant. Sie gilt für alle lagerungsarten des Stabes. In diesem Falle kann sie geschlossen gelöst werden. In [1], [2] und [9] sind für einige wichtige Belastungsfälle und Randbedingungen geschlossene Lösungen dieser Differentialgleichung angegeben.

Sind die Koeffizienten $\operatorname{EI}_{\omega \omega, D}(z)$ und $G K(z)$ beliebige Funktionen von $z$, dann ist im allgemeinen eine geschlossene Lösung nicht mehr möglich. Die Differentialgleichung (18) muss dann numerisch gelöst werden, indem das Problem diskretisiert wird. Dies kann mathematisch oder physikalisch sein. Die mathematische Diskretisierung des Problems (Differenzenmethode) wird hier nicht behandelt. Im folgenden wird ein Modell entwickelt, das eine beliebig genaue Lösung der Differentialgleichung (18) ermöglicht. Das Modell ist eine physikalische Diskretisierung des Torsionsproblems.

\subsection{Model1}

Die Differentialgleichung (18) kann folgendermassen interpretiert werden. Ein dünwandiger Stab bringt der Torsionsbelastung zwei Widerstände entgegen: erstens durch Bildung eines geschlossenen Schubflusses in der Querschnittswandung (St. Venantsche Torsion) und zweitens durch Schubspannungen, die aus der Aenderung des Wölbmomentes in stablängsrichtung entstehen (Wölbtorsion). Das Wölbmoment ist eine aus Normalspannungen gebildete Gleichgewichtsgruppe. Je grösser der eine Widerstand gegenüber dem anderen ist, um so grösser ist auch sein Anteil am gesamten Torsionsmoment im Querschnitt. Man kann sich also den Stab so vorstellen, wie wenn er aus zwei gleich langen Teilstäben zusammengesetzt wäre, die je einen der beiden Teilwiderstände aufweisen und miteinander durch unendlich viele Bindungen verbunden sind (Bild 4a). 


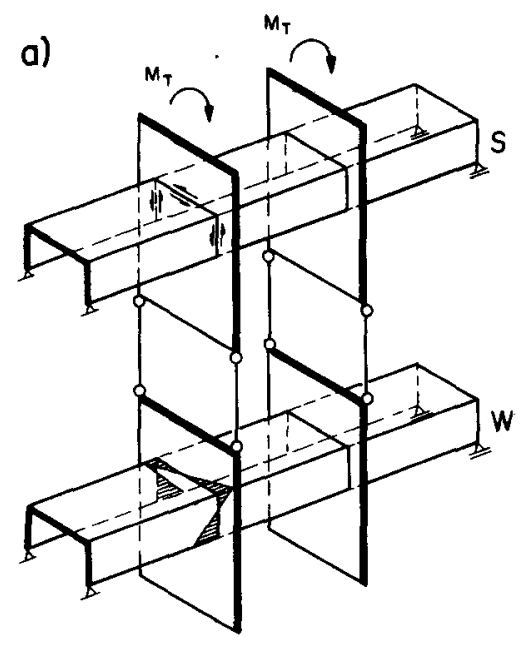

b)

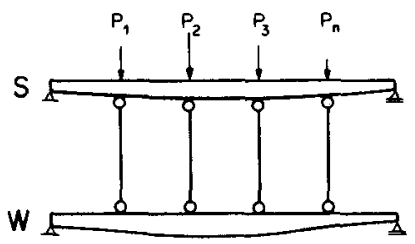

Bild 4 Trägermodell a) und seine Wirkungsweise b)

Die Wirkungsweise soll in Bild 4b mit zwei Biegeträgem unterschiedlicher Steifigkeit illustriert werden. Die beiden Träger sind durch starre Pendelstäbe miteinander verbunden und nehmen je nach Steifigkeit einen Teil der Belastung auf. Im Modell des Torsionsträgers entspricht der Stab $S$ dem Träger mit nur St. Venantscher Torsionssteifigkeit GK, der Stab W demjenigen mit nur Wölbsteifigkeit $E I_{w \omega}$. Die Durchbiegungen der beiden Stäbe, die bei den Bindungen gleich gross sind, entsprechen den Stabverdrehungen und die Kräfte $P_{i}$ äusseren Drehmomenten $\mathbb{M}_{T}$

Für die numerische Berechnung wird eine endliche Zahl von Bindungen angenommen (Bild 4a). Je mehr Bindungen vorhanden sind, desto genauer ist das Resultat. Die Berechnung wird analog zum Biegeträger durchgeführt, bei dem die Kräfte in den Pendelstäben als Jeberzählige eingeführt werden. 

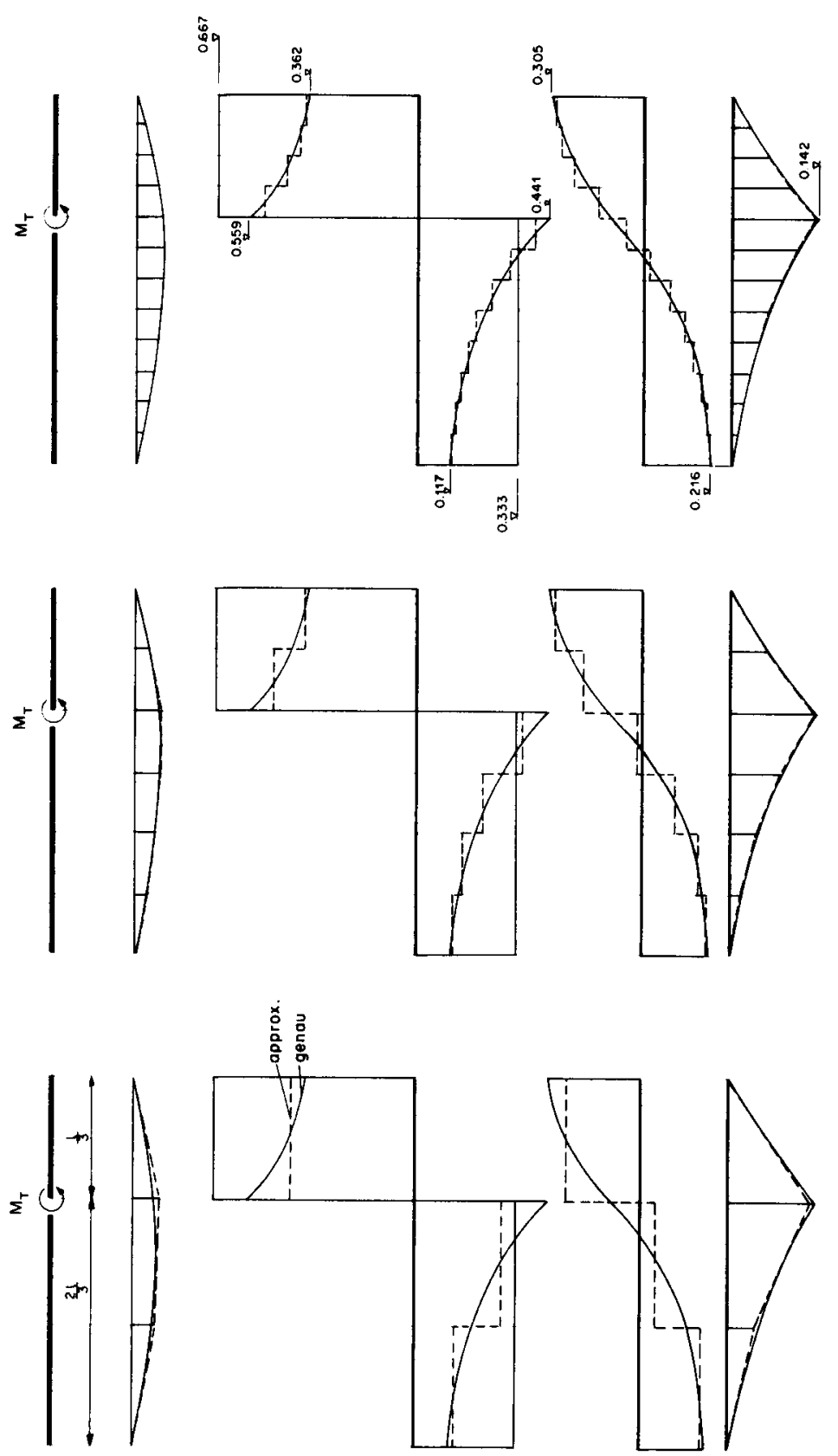
Schon mit wenigen Bindungen können die beiden Torsionsanteile recht gut abgeschätzt werden. Bild 5 zeigt die Berechnung eines durch ein äusseres Drehmoment belasteten, prismatischen Trägers mit 2,5 und 11 Bindungen. Der $x$-Wert des Trägers beträgt:

$$
x=\sqrt{G K l^{2} / E I_{\omega \omega}}=3
$$

Die Lösung des Torsionsproblems mit dem angegebenen Modell nach dem Kraftgrössenverfahren erfordert das Aufstellen von linearen Gleichungssystemen und deren Lösung. Die Berechnung der Matrixkoeffizienten ist eine immer wiederkehrende Operation, die einfach programmiert werden kann. Zur Lösung der Gleichungssysteme sind zahlreiche Bibliotheksprogramme vorhanden. Das in Bild 5 dargestellte Beispiel wurde mit Hilfe eines ALGOL - Programms gerechnet.

In letzter Zeit verbreitet sich STRESS, eine problemorientierte Computersprache zur Berechnung von Stabtragwerken, in der Bauingenieurpraxis [10]. Da das Modell des Torsionsträgers ein Stabtragwerk ist, kann dieses Programm auch zur Lösung des Torsionsproblems verwendet werden. Dazu muss jedoch das Modell abgeändert werden.

Im STRESS - Programm wird nur die St. Venantsche Torsionssteifigkeit der Stäbe berücksichtigt. Die vollständige Analogie zwischen Biegung und Verdrehung ermöglicht es auch, die Wölbsteifigkeit zu erfassen:

$$
\begin{array}{ll}
\text { Diff.-gleichung für Biegung } & :\left(E I_{y y} \eta^{\prime \prime}\right)^{\prime \prime}=\mathrm{p}_{\mathrm{y}} \\
\text { Diff.-gleichung für Torsion } \quad(G K=0):\left(E I_{w \omega} \varphi^{\prime \prime}\right)^{\prime \prime}=\mathrm{m}_{\mathrm{T}}
\end{array}
$$

Ein Torsionsträger mit der Wölbsteifigkeit $E I_{\omega \omega}$ kann demnach durch einen Biegeträger mit der Biegesteifigkeit $E I_{y y}$ simuliert werden. Die Durchbiegung $\eta$ und die äussere Belastung $p_{y}$ des Biegeträgers entsprechen der Verdrehung $\varphi$ und dem äusseren Drehmoment $\mathrm{m}_{\mathrm{T}}$ des Torsionsstabes. 
Im Trägermodell für die Berechnung mit STRESS (Bild 6) ist der Stab $S$ ein Torsionsträger mit der St. Venantschen Torsionssteifigkeit GK und der Stab W ein Biegeträger mit der Biegesteifigkeit $\mathrm{EI}_{\omega \omega}$. Das einfachste, aus biege- und torsionssteifen stäben bestehende System, das mit STRESS behandelt werden kann, ist ein ebener Trägerrost. Deshalb ist das Modell des Torsionsträgers ein Trägerrost bestehend aus den Stäben $S$ und $W$, die mit starren Querträgerm von der Einheitslänge miteinander verbunden werden. Die Durchbiegung des Trägers W, die der Verdrehung des Stabes mit Wölbsteifigkeit entspricht, ist gleich der Verdrehung des Stabes $S$. Die äussere Belastung $M_{T}$ wird entweder am Stab $S$ als Drehmoment oder am Stab $W$ als Vertikalkraft eingeführt.

In Bild 7 sind zwei mögliche Modelle für einen durchlaufenden, prismatischen Stab angegeben, die mit STRESS berechnet werden sollen. Die Einschränkung, dass der Stab prismatisch sei, ist deshalb notwendig, weil die Differentialgleichung (18) fü durchlaufende Stäbe mit variablem Querschnitt nicht gültig ist, sonderm im allgemeinen nur eine gute Näherung darstellt. Im sechsten Kapitel wird dieses Problem behandelt.

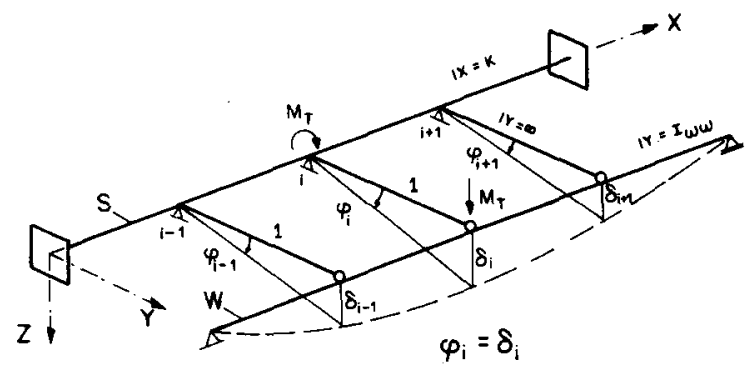

Bild 6 Modell des Torsionsträgers für die Berechnung mit STRESS (Koordinatensystem und Stabwerte nach STRESS)

Das statische System und die äussere Belastung sind in Bild 7a angegeben. Der Träger ist am linken Ende gegen Biegung und Verdrehung eingespannt. Da die Biegesteifigkeit IY des Stabes $S$ je nach lagerung der Querträger beliebig oder $\infty$ gross sein kann, ist 
es gleichgültig, ob dieser für $\varphi=0$ total eingespannt ist oder nur gegen Verdrehen gehalten wird. Einfachheitshalber wird bei. STRESS die totale Einspannung gewählt. Beim Stab W spielt es keine Rolle, ob er gegen Verdrehen eingespannt ist oder nicht, weil

a)

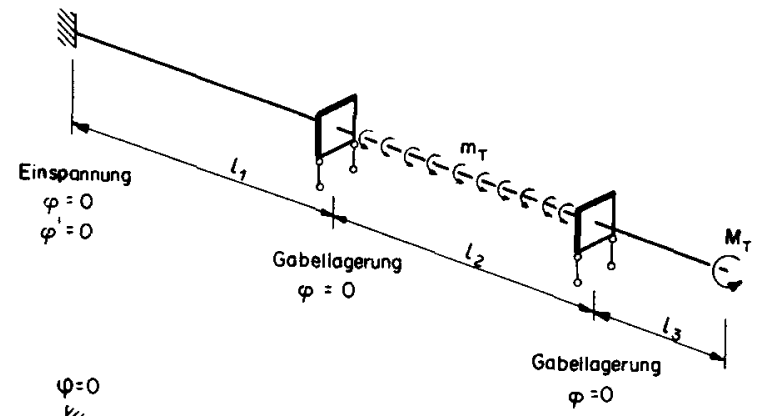

b)
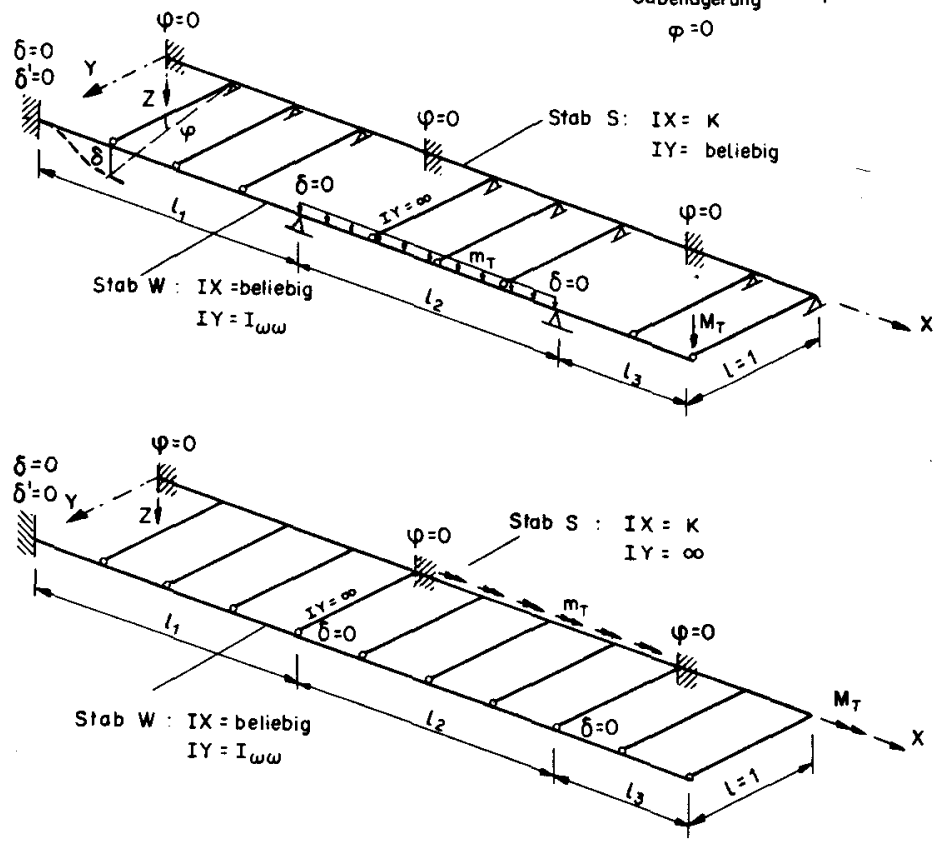

Bild 7 Berechnung eines durchlaufenden, prismatischen Stabes unter äusserer Torsionsbelastung mit STRESS

a) Statisches System und Belastungen

b) Zwei mögliche Modelle 
seine Torsionssteifigkeit IX beliebig sein kann, und weil er nur durch vertikale Lasten belastet wird. Die Gabellagerung bedeutet für Stab $S$ wiederum eine Einspannung und für Stab $W$ ein um die Y-Achse frei drehbares Lager, welches durch einen starren Querträger ersetzt werden kann (Bild 7b). Die Querträger mit IY $=\infty$ können an den Stab $\mathbb{W}$ auch biegesteif angeschlossen werden. In diesem Fall müssen die Torsionssteifigkeiten der Querträger und des Stabes $W \infty$ klein sein. ( $\infty$ gross oder klein bedeutet bei der Verwendung von STRESS sehr gross oder sehr klein gegenüber anderen Stabwerten). Die Belastung kann entweder als Torsionsmoment am Stab S oder als vertikale Kraft am Stab W eingeführt werden.

Auch elastische Torsionseinspannungen können berücksichtigt werden. In diesem Fall wird das untere Modell verwendet. Die elastische Einspannung wird dadurch simuliert, indem an der Einspannstelle des Stabes $S$ ein neuer Querträger entsprechender Biegesteifigkeit in Richtung der negativen Y-Achse angebracht, und dieser Knoten in Z-Richtung unterstuitzt wird. 
4. VERNACHLAESSIGUNG DER ST. VENANTSCHEN TORSION

Oft ist die St. Venantsche Torsionssteifigkeit GK vernachlässigbar klein. Die Gleichung (18) nimmt dann folgende Form an:

$$
\left(E I_{\omega \omega} \varphi^{\prime \prime}\right)^{\prime \prime}=\mathrm{m}_{\mathrm{T}}
$$

Dabei wird von jetzt an für $I_{\omega \omega, D}$ einfachheitshalber $I_{w \omega}$ gesetzt. In diesem Fall ist die Berechnung besonders einfach. Wegen der formellen Analogie der Differentialgleichung (19) mit derjenigen des auf Biegung beanspruchten Stabes, können alle bekannten Verfahren und Tabellenwerke des Biegeträgers zur Lösung des Torsionsproblems verwendet werden.

Aus der Herleitung der Differentialgleichungen der Torsion (Kapitel 3) ist ersichtlich, dass der Term (GK $\varphi^{\prime}$ ) in Gl. (18) unabhängig von der Lagerung des Stabes ist. Das unterschiedliche Torsionsverhalten von Stäben mit prismatischen und variablen Querschnitten rürt somit nur vom Anteil der Wölbtorsion her.

Im folgenden wird der Stab mit $G K=0$ untersucht, mit dessen Hilfe gezeigt werden soll, worin der Unterschied besteht, und wie beliebig gelagerte stäbe berechnet werden können.

\subsection{Verhalten unter Torsion}

Der Unterschied in Verhalten prismatischer Stäbe und solcher mit variablen Querschnitten soll am Beispiel eines Brïckenträgers mit einfachsymmetrischem Dreischeibenquerschnitt demonstriert werden.

Bild 8a zeigt einen prismatischen Stab mit Gabellagerung an beiden Enden unter Torsionsbelastung. Die Wölbspannungen, die mit G1. (19) gerechnet werden können, sind in jedem Querschnitt einander ähnlich, so dass die Spannungsnullpunkte auf drei zur stab- 
achse parallelen Geraden liegen. Da diese drei spannungsfreien Fasern nach der Belastung ihre Länge nicht änderm, bleiben ihre Fndpunkte in der ursprünglichen Lage (Bild 8b).

a)

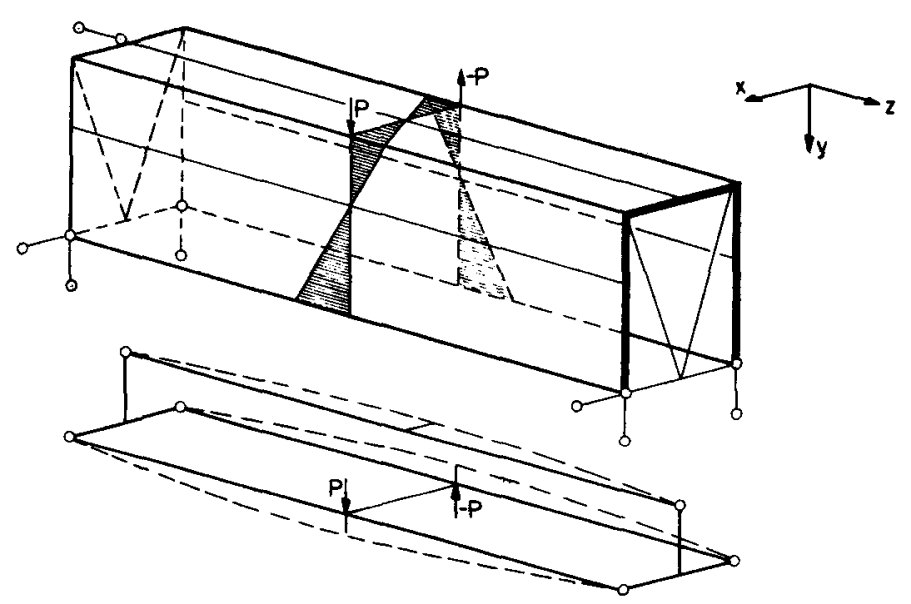

c)
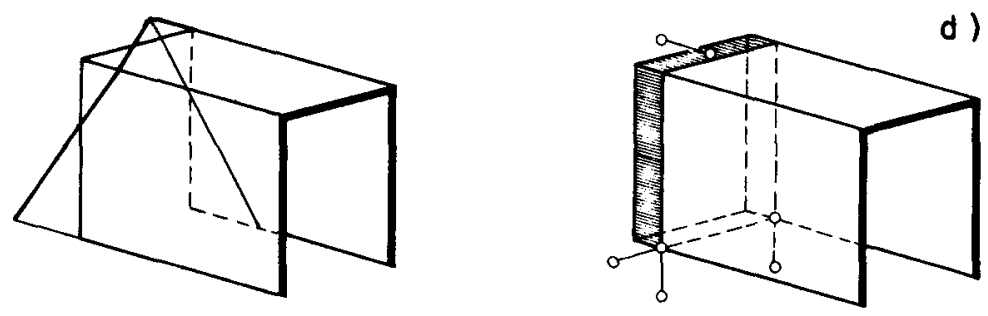

Bild 8 Torsionsverhalten des prismatischen Stabes

a) Spannumgsverteilung im Querschnitt

b) Die Spannungsnullpunkte liegen auf 3 Geraden, deren Endpunkte nach der Deformation nicht verschoben werden

c) Verwölbung des freien Endquerschnitts aus der Ebene

d) Starrer Fndblock bleibt in der ursprünglichen Lage 
Der Stabendquerschnitt verwölbt sich aus der durch diese drei Punkte bestimmten Ebene heraus (Bild 8c).

Im Falle des Trägers mit veränderlicher Höhe bilden die Spannungsnullpunkte der Seitenwände einen Bogen, dessen Enapunkte sich unter der Belastung verschieben (Bild 9a,b). Aus Symmetriegründen bleibt die spannungsfreie Faser der mittleren Scheibe gerade, und

a)

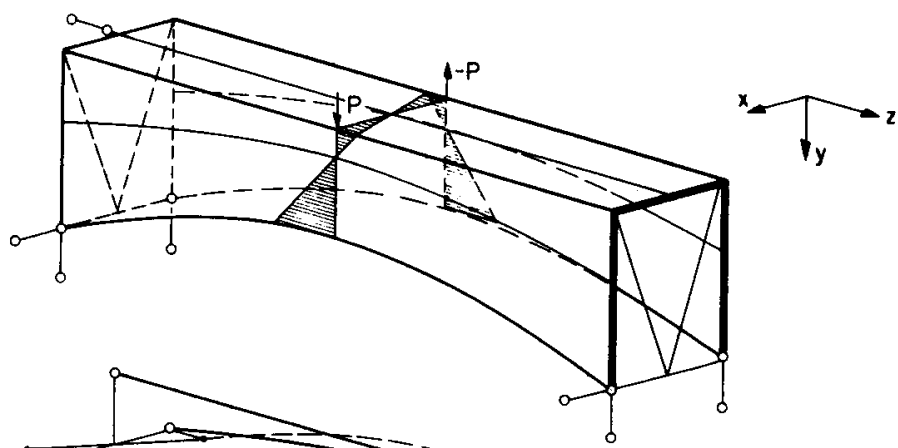

b)

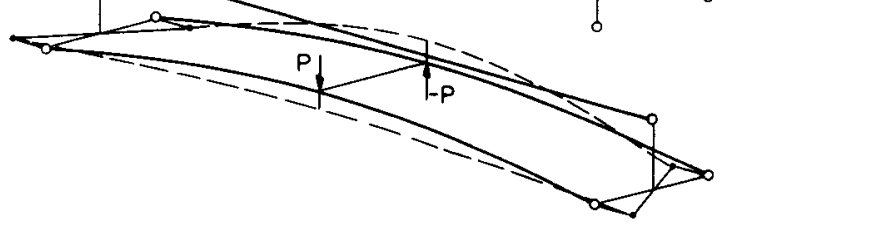

c)
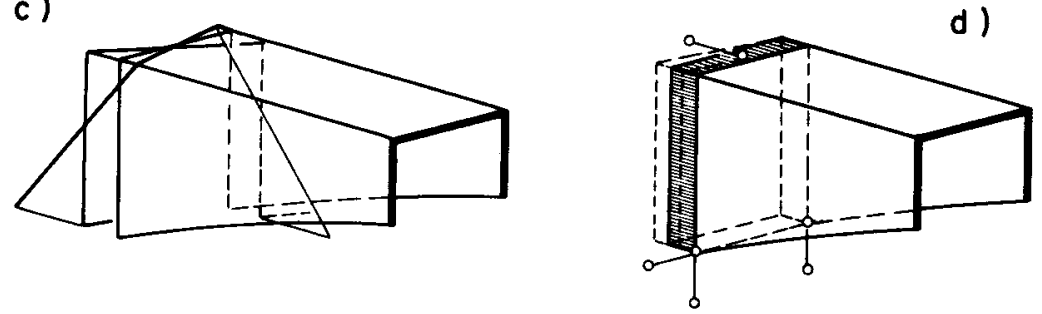

Bild 9 Torsionsverhalten des Stabes mit veränderlichem Querschnitt

a) Spannungsverteilung im Querschnitt

b) Die Spannungsnullpunkte der Seitenwände bilden einen Bogen, deren Fndpunkte verschoben werden

c) Verwölbung des freien Endquerschnittes aus der verdrehten Querschnittsebene

d) Starrer Endblock verareht sich 
ihre Fndpunkte verändern die ursprüngliche Lage nicht. Der Stabendquerschnitt verwölbt sich aus der durch diese drei Endpunkte gebildeten Vertikalebene heraus, die sich um die y-Achse dreht (Bild 9c). Der Endquerschnitt erfährt somit neben der Verwölbung auch eine Verdrehung. So lange er sich ungehindert verwölben und verdrehen kann, wie es durch die in Bild 9a dargestellte Lagerung möglich ist, entstehen in den Stabquerschnitten nur Wölbmomente und keine Biegemomente. Deshalb hat die Gleichung (19) auch für diesen Fall Gïltigkeit.

Wird der Träger an eineri Ende eingespannt, so bedeutet das für den prismatischen Stab eine Wölbbehinderung. Es entstehen an dieser Einspannstelle Wölbmomente. Beim Träger mit variabler Höhe erzeugt die Einspannung neben Wölbmomenten auch Biegemomente $\mathrm{M}_{\mathrm{X}}$, weil die Verdrehung verhindert wird.

Wenn an den Stabenden statt biegsame Scheiben starre Fndblöcke angeordnet werden, die Lagerung jedoch beibehalten wird (Bild $8 d$ und 9d), so wird in beiden Fällen eine wölbbehinderung erreicht, d.h. eine Einspannung gegen die Verwölbung. Während der starre Endblock des prismatischen stabes unter äusserer Torsionsbelastung an Ort und Stelle bleibt, verdreht sich derjenige des stabes mit variablem Querschnitt um die vertikale y-Achse. Auch für diesen Fall ist die Torsionsdifferentialgleichung (19) guiltig. Wird die Verdrehung des Endblockes durch Einführung eines Biegemomentes verhindert, so verursacht, wie später gezeigt wird, dieses Biegemoment im Stab Wölbmomente, die ihrerseits wieder Verdrehungen erzeugen. Diese gegenseitige Beeinflussung von Biegung und Verdrehung wird durch das im Kapitel 3 hergeleitete Differentialgleichungssystem (9) beschrieben.

Beim Träger mit einfachsymmetrischem, variablem Querschnitt bleiben die Querschnittspunkte auf der Symmetrieachse unter Torsionsbelastung spannungsfrei. Da diese Punkte auf einer Geraden liegen, bleiben deren Endpunkte in ihrer ursprïnglichen Lage, und der Endquerschnitt verdreht sich um die Symmetrieachse. Wenn die 
Querschnitte unsymmetrisch sind, so verdreht sich die Ebene, die durch die spannungsfreien Punkte des Endquerschnittes gebildet wird, um beide Querschnittsachsen.

Auch Stäbe mit mehr als drei Scheiben zeigen dasselbe Verhalten. Da die Wölbnormalspannungen im Querschnitt infolge eines Wölbmomentes nur von der Geometrie des Querschnittes abhängen, ist seine Verwölbung aus der ursprünglichen Ebene unabhängig davon, ob er einem prismatischen Stab angehört oder nicht (Voraussetzung sieben). Bei nichtprismatischen Stäben tritt neben der Verwölbung auch eine Verdrehung der ursprünglichen Querschnittsebene auf, weil die spannungsfreien Punkte nicht auf Geraden liegen.

\subsection{Beliebige Lagerung}

Weist der Stab eine beliebige Lagerung auf, so kann man ihn durch Entfernen von genügend vielen Bindungen auf die oben behandelten Grundsysteme zuruickführen, so dass die Differentialgleichung (19) zur Anwendung kommt. Durch die Einführung der gelösten Lagerreaktionen als iberzählige Grössen, und ihre Bestimmung aus den Elastizitätsbedingungen werden die Schnittkräfte des gegebenen Systems aus Ueberlagerung der Einflüsse ermittelt. Die Berechnung soll zunächst an einem prismatischen Stab durchgeführt werden.

Der wölbspannungsfreie Endquerschnitt eines prismatischen Trägers sei im Querschnittspunkt 3 in Stablängsrichtung festgehalten (Bild IOa). Die Reduktion der Festhaltekraft $X_{1}$ auf das feste Lager in 1 erzeugt neben der Kraftkomponente, die vom Lager aufgenommen wird, ein Kräftepaar $X_{1} d$ in der Ebene $E$ (Bild 10b). Der Träger wird somit am linken Ende durch ein Biegemoment $\mathbb{M}=-X_{1} d$ belastet. Der Verlauf des Biegemomentes in der Ebene $E$ ist aus Bild lob ersichtlich. In einem allgemeinen Querschnitt können die drei Schnittkräfte $\mathbb{M}_{x}, \mathbb{M}_{y}, \mathbb{M}_{\omega}$ durch ein äquivalentes Iängskräftepaar dargestellt werden, welches in den zueinander parallelen und in der Ebene $E$ liegenden Stabfasern durch die Punkte 1 und 3 angreift. Diese in z-Richtung linear veränderlichen Längskräfte $\mathrm{X}_{1}(\mathrm{z})$ erzeugen neben dem Biegemoment auch Wölbmomente. Aus der Definition des Wölbmomentes 
a)

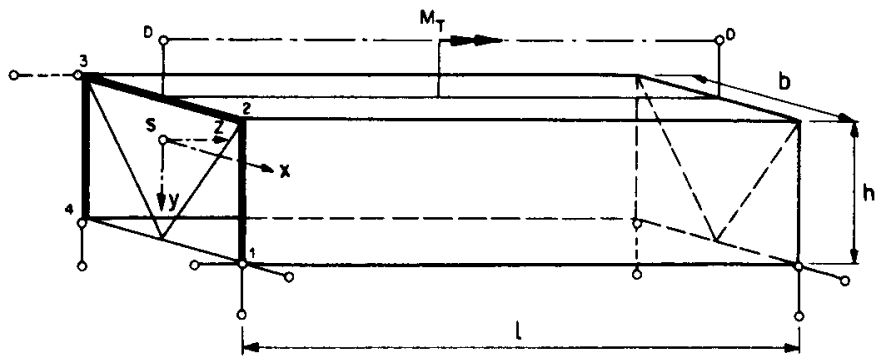

b )

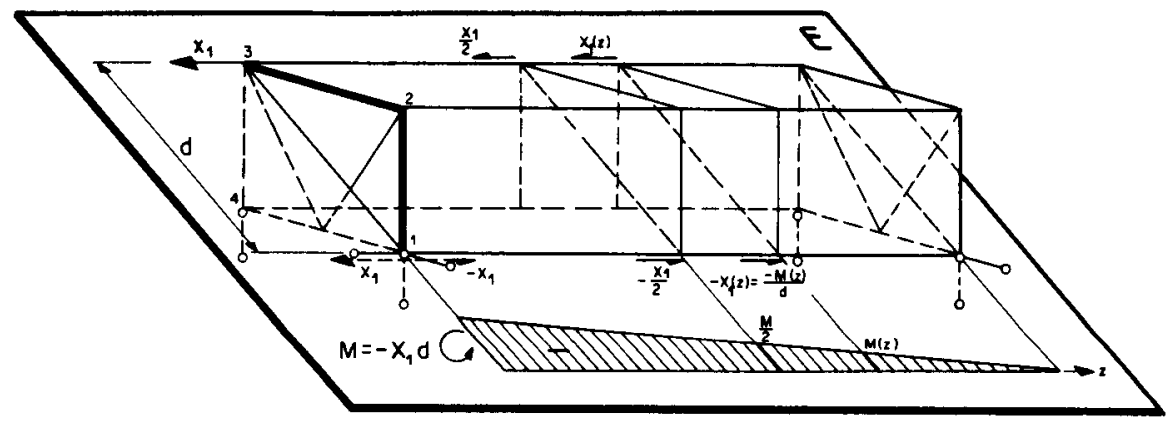

c)

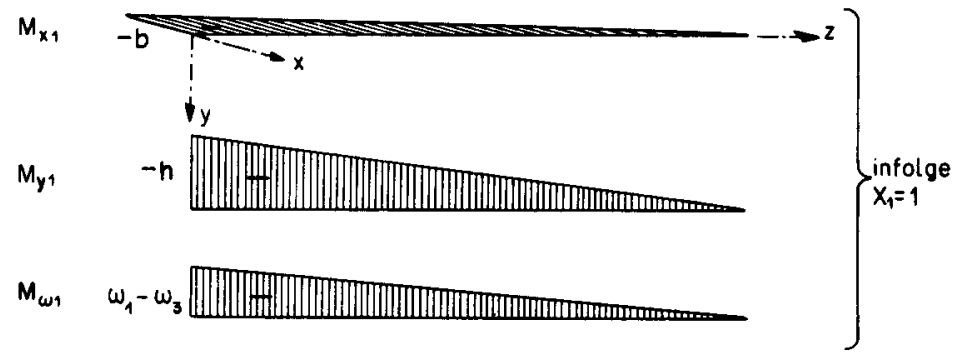

$M_{\omega 0}$

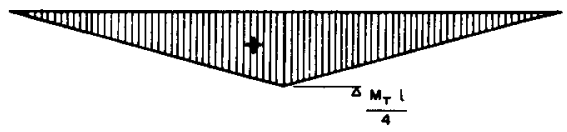

Bild 10 Beliebig gelagerter Stab

a) System und Belastungen

b) Grundsystem mit der Ueberzähligen $X_{1}$

c) Schnittkräfte am Grundsystem 


$$
M_{\omega}=\int_{\tilde{F}} \sigma \omega d \mathrm{~F}
$$

folgt bei längskräften $\mathrm{P}_{i}$, die in einem beliebigen Querschnitt angreifen,

$$
\mathrm{M}_{\omega}=\sum_{i} \mathrm{P}_{\mathrm{i}} \omega_{\mathrm{i}}
$$

Das Wölbmoment im Querschnitt $z$ infolge der Längskräfte $X_{\mathbb{A}}(z)$ und - $X_{1}(z)$ ist somit

$$
M_{\omega}(z)=X_{1}(z)\left(\omega_{1}-\omega_{3}\right)
$$

Hierin bedeuten $\omega_{1}$ und $\omega_{3}$ die sektoriellen Koordinaten der Punkte 1 und 3 im Querschnitt $\mathbf{z}$.

Aus Bild $10 c$ gehen die Verläufe der Biegemomente $M_{x}, M_{y}$ und des Wölbmomentes $\mathrm{M}_{\omega}$ infolge der Ueberzähligen $\mathrm{x}_{1}=1$ hervor. Im weiteren ist das Wölbmoment $M_{\omega 0}$ infolge einer äusseren Belastung $\mathbb{M}_{T}$ gezeigt. Aus diesen Momenten kann die Verschiebung des Punktes 3 am Grundsystem berechnet werden.

Das äussere Drehmoment verursacht die Verschiebung $\delta_{10}$ des Punktes 3 aus seiner ursprünglichen Ebene.

$$
\delta_{10}=\int_{0}^{\ell} \frac{\mathrm{M}_{\omega} \mathrm{M}_{\omega}^{0}}{\mathrm{EI} I_{\omega \omega}} \mathrm{dz}
$$

Bie Verschiebung infolge $x_{1}=1$ lautet:

$$
\delta_{41}=\int \frac{M_{x}^{2}}{E I_{x x}} d z+\int \frac{M_{y}^{2}}{E I_{y y}} d z+\int \frac{M_{\omega}^{2}}{E I_{\omega \omega}} d z
$$

Mit der Elastizitätsbedingung

$$
\mathrm{X}_{1} \delta_{H}+\delta_{10}=0
$$


lautet die unbekannte Festhaltekraft:

$$
\mathrm{X}_{1}=-\delta_{10} / \delta_{11}
$$

Wenn auf diese Art mindestens vier Querschnittspunkte festgehalten werden, so bedeutet das eine Einspannung des Stabes sowohl gegen Verwölbung als auch gegen Verdrehung um die Querschnittshauptachsen. Dabei müssen diese vier Punkte so gewählt werden, dass sie nach der Verwölbung nicht in einer Ebene liegen. Bei prismatischen Stäben, die durch äussere Drehmomente belastet werden, erzeugen diese vier Festhaltekräfte an der Einspannstelle nur ein Wölbmoment und keine Biegemomente, d.h. sie bilden eine Gleichgewichtsgruppe.

Analog können auch Träger mit veränderlichem Querschnitt behandelt werden, indem die Querschnitte als stickweise konstant angenommen werden, Dabei ist zu beachten, dass die Längskräftepaare links und rechts des Querschnittsprunges verschiedene Wölbmomente erzeugen. Bei unsymmetrischen Querschnitten wird das Biegemoment in verschiedene Ebenen zerlegt.

Die Berechnung soll an einem einfachen Beispiel durchgeführt werden. In Bild lla ist ein aus zwei gleich langen Stucken bestehender Träger mit einfachsymmetrischem Querschnitt dargestellt, der in Stabmitte durch ein konzentriertes Torsionsmoment $\mathrm{M}_{\mathrm{T}}=1 \mathrm{mt}$ belastet wird. Am linken Ende sei er total eingespannt und am rechten Ende gabelartig gelagert. Das Grundsystem und die Querschnittswerte der beiden Teilquerschnitte gehen aus Bild llb und c hervor.

Um die volle Einspannung des Trägers am linken Fnde gegen Verdrehung und Verwölbung zu realisieren, muss der Endquerschnitt mindestens in vier Punkten festgehalten werden. Neben dem festen Lager 1 werden in drei weiteren Querschnittspunkten Bindungen angebracht. In Bild 12 sind alle Schnittkräfte des Grundsystems eingetragen, die von den Ueberzähligen und der äusseren Belastung herrühren. 
a)

b)
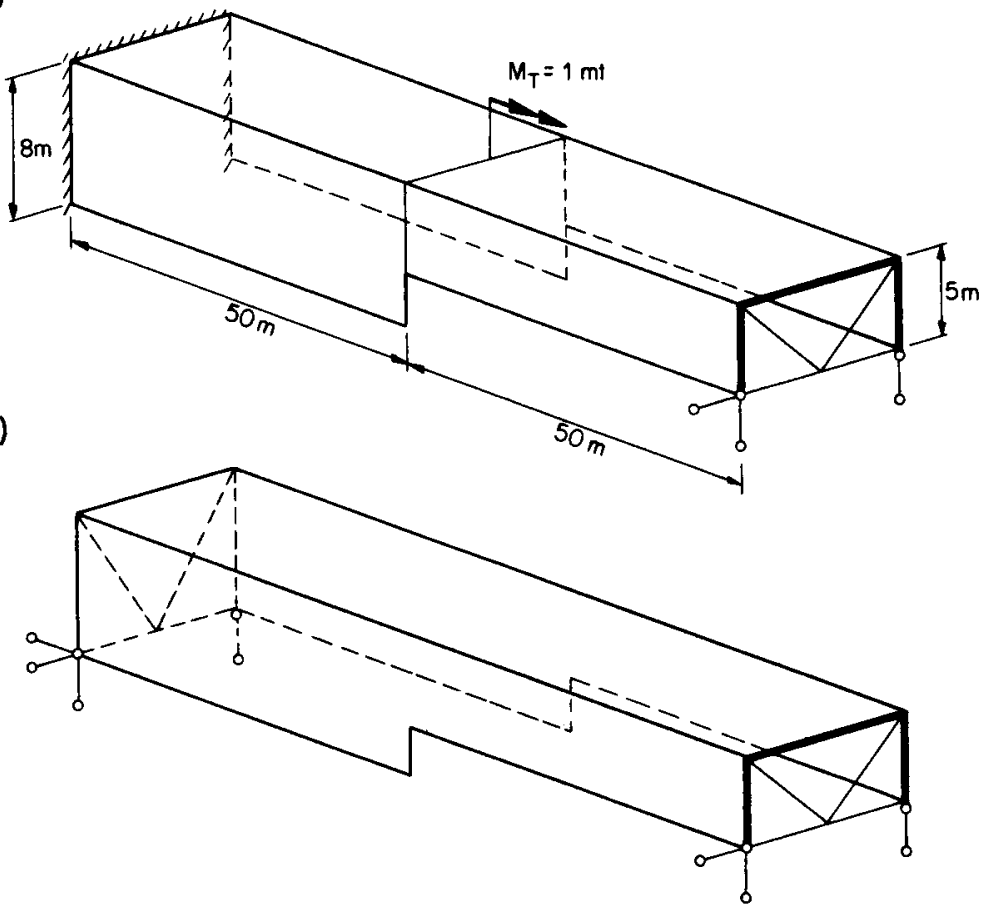

c)

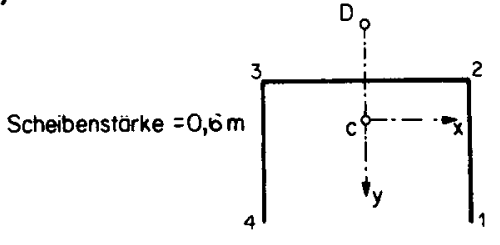

Querschnitt 1

\begin{tabular}{|c|c|c|}
\hline IK & $=$ & $2,0160 \mathrm{~m}^{4}$ \\
\hline$I_{\omega \omega}$ & $=$ & $2949,1200 \mathrm{~m}^{6}$ \\
\hline$I_{x x}$ & $=$ & $432,2880 \mathrm{~m}^{4}$ \\
\hline$I_{y y}$ & $=$ & $117,2446 \mathrm{~m}$ \\
\hline$\omega_{1}$ & $=$ & $28,8000 \mathrm{~m}^{2}$ \\
\hline$\omega_{2}$ & $=$ & $-19,2000 m^{2}$ \\
\hline$\omega_{k}$ & $=$ & \\
\hline
\end{tabular}

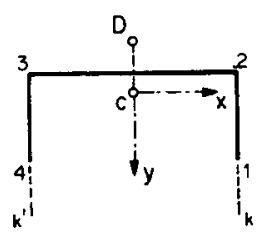

Querschnitt 2

$\begin{aligned} 1,5840 & \mathrm{~m}^{4} \\ 835,7143 & \mathrm{~m}^{6} \\ 302,5800 & \mathrm{~m}^{4} \\ 33,1705 & \mathrm{~m}^{4} \\ 19,2857 & \mathrm{~m}^{2} \\ -10,7143 & \mathrm{~m}^{2} \\ 37,2857 & \mathrm{~m}^{2}\end{aligned}$

Bild 11 Träger mit stückweise konstantem Querschnitt
a) System und Belastung
b) Grundsystem
c) Querschnittwerte der beiden Teilquerschnitte 


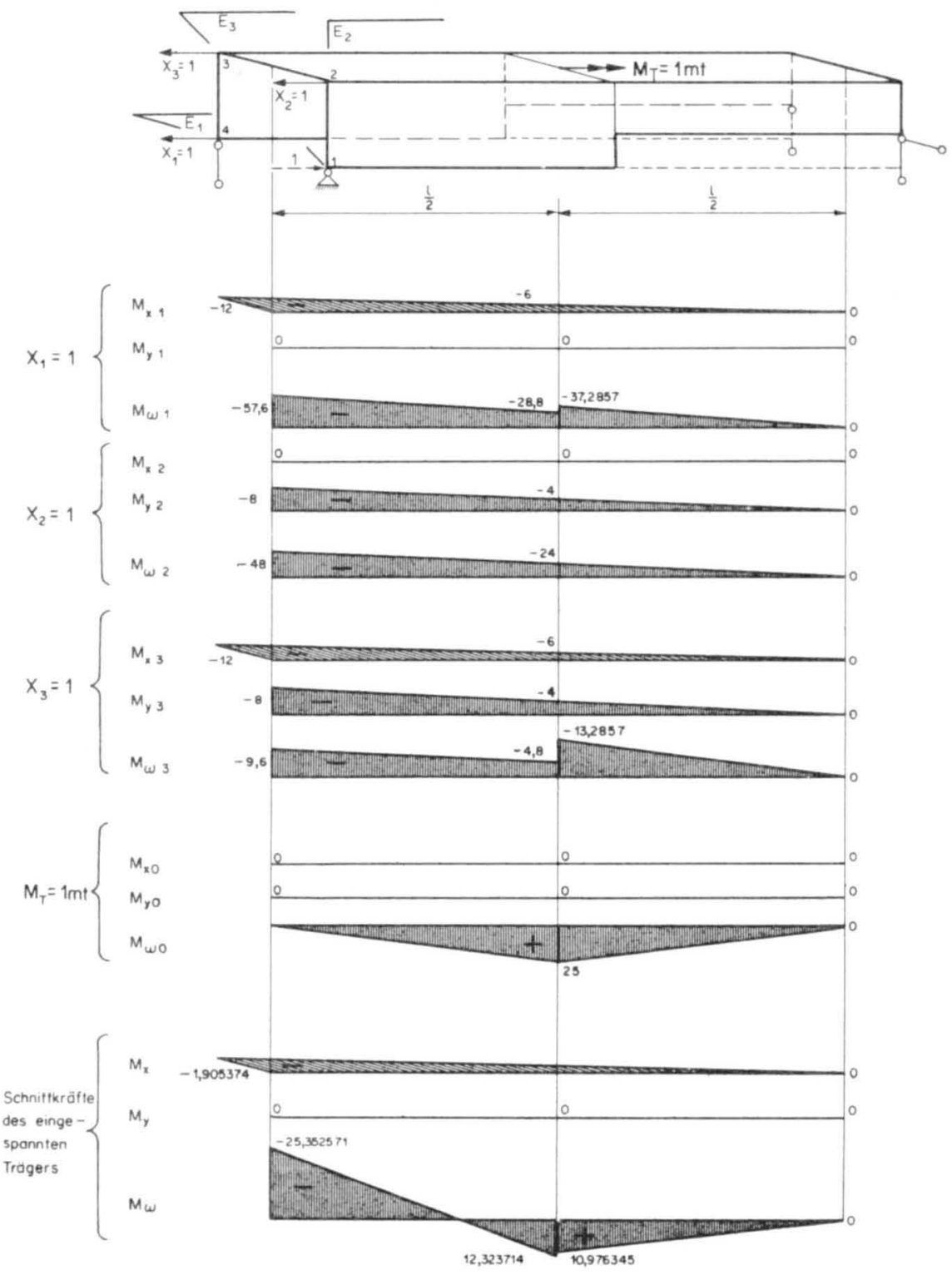

Bild 12 Schnittkräfte des Grundsystems und des eingespannten Trägers bei drei Ueberzähligen (ohne Ausnützung der Symmetrie) 
Die von den drei Ueberzähligen $X_{1}, X_{2}$ und $X_{3}$ erzeugten Längskräftepaare wirken in den Ebenen $E_{1}, E_{2}$ und $E_{3}$. Wenn die Normalkraft ausserhalb des Stabquerschnittes angreift, wie es beim zweiten Segment für die durch die Punkte 1 und 4 gehenden Faserm der Fall ist, so wird angenommen, dass diese Faserm durch starre Konsolen mit dem Querschnitt verbunden sind. Die dazugehörigen Wölbkoordinaten der Punkte $k$ und $k^{\prime}$ sind aus Bild $11 \mathrm{c}$ ersichtlich.

Die Elastizitätsbedingungen erfordem, dass die Verschiebungen der Punkte 2, 3 und 4 aus ihrer ursprünglichen Lage verschwinden sollen.

Die Verschiebungsgrössen $\delta_{i k}$ lauten:

$$
\delta_{i k}=\int_{0}^{\ell} \frac{M_{x i} \mathbb{M}_{x k}}{E I_{x x}} d z+\int_{0}^{\ell} \frac{M_{y i} \mathbb{M}_{y k}}{E I_{y y}} d z+\int_{0}^{\ell} \frac{M_{\omega i} \mathbb{M}_{\omega k}}{E I_{\omega \omega}} d z
$$

Die E-fachen Verschiebungsgrössen sind in der folgenden Tabelle I zusammengestellt. Um die gute Uebereinstimmung der Resultate mit der späteren Berechnung zu zeigen, werden alle Zahlen sechsstellig angegeben. Die sechste Stelle nach dem Komma ist gerundet.

Die Lösung des Gleichungssystems

$$
\begin{aligned}
& \mathrm{X}_{1} \delta_{11}+\mathrm{X}_{2} \delta_{12}+\mathrm{X}_{3} \delta_{13}+\delta_{10}=0 \\
& \mathrm{X}_{1} \delta_{21}+\mathrm{X}_{2} \delta_{22}+\mathrm{X}_{3} \delta_{23}+\delta_{20}=0 \\
& \mathrm{X}_{1} \delta_{31}+\mathrm{X}_{2} \delta_{32}+\mathrm{X}_{3} \delta_{33}+\delta_{30}=0
\end{aligned}
$$

ist:

$$
\begin{aligned}
& x_{1}=0,327602 t \\
& x_{2}=0,168821 t \\
& x_{3}=-0,168821 t
\end{aligned}
$$


Tabelle 1

$E \delta_{i k}=\int_{0}^{\ell} \frac{M_{x i} M_{x k}}{I_{x x}} d z+\int_{0}^{\ell} \frac{M_{y i} M_{y k}}{I_{y y}} d z+\int_{0}^{\ell} \frac{M_{\omega i} M_{\omega k}}{I_{\omega \omega}} d z$

\begin{tabular}{|c|c|c|c|c|c|c|c|}
\hline$\delta_{11}=$ & 11,698692 & + & 0 & + & 60,537753 & $=$ & 72,236445 \\
\hline $\mathrm{E} \delta_{12}=$ & 0 & 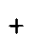 & 0 & + & 45,189897 & $=$ & 45,189897 \\
\hline $\mathrm{E} \delta_{13}=$ & 11,698692 & + & 0 & + & 15,347856 & $=$ & 27,046548 \\
\hline $\mathrm{E} \delta_{22}=$ & 0 & + & 23,960403 & + & 34,273638 & $=$ & 58,23404 \\
\hline $\mathrm{E} \delta_{23}=$ & 0 & + & 23,960403 & + & 10,916259 & $=$ & 34,876662 \\
\hline$\delta_{33}=$ & 11,698692 & + & 23,960403 & ${ }^{\top}$ & 4,431597 & $=$ & 40,090692 \\
\hline $\mathrm{E} \delta_{10}=$ & 0 & + & 0 & - & 26,727757 & $=$ & $-26,72775$ \\
\hline $\mathrm{E} \quad \delta_{20}=$ & 0 & + & 0 & - & 18,747496 & $=$ & - 18,747 \\
\hline $30=$ & 0 & + & 0 & - & 7,980261 & $=$ & $-\quad 7,9$ \\
\hline
\end{tabular}

Die endgiltigen Schnittkräfte gehen ebenfalls aus Bild 12 hervor. Da es sich um einen Stab mit einfachsymmetrischem Querschnitt handelt, entstehen neben Wölbmomenten $M_{\omega}$ nur Biegemomente $M_{x}$ um die vertikale Symmetrieachse. Die Momente an der Einspannstelle sind:

$$
\begin{aligned}
& M_{\omega}=-25,352571 \mathrm{~m}^{2} \mathrm{t} \\
& \mathrm{M}_{x}=-1,905374 \mathrm{mt}
\end{aligned}
$$

Im Spezialfall des einfachsymmetrischen Querschnittes ist es sinnvoller, das Wölbmoment $\mathrm{M}_{\omega}$ und das Kräftepaar $\mathrm{X}_{1} \mathrm{~b}$ an der Einspannstelle als Ueberzählige einzuführen. Dabei ist es für das Endresultat gleichgültig, in welcher Horizontalebene das Kräftepaar angenommen wird.

Die Berechnung soll für den gleichen Träger mit den neuen Ueberzähligen durchgefihrt werden. Die Horizontalebene des Biegemomentes $\mathbb{M}_{X}$ wird durch diejenigen Punkte des ersten Querschnittes gelegt, für welche die Wölbkoordinate den Wert Null besitzt (Bild 13). Somit erzeugt das Biegemoment $M_{x}=-X_{1} b$ nur im zweiten segment Wölbmomente. 
Mit den in Tabelle 2 zusammengestellten E-fachen Verschiebungsgrössen werden die Ueberzähligen $\mathrm{X}_{1}$ und $\mathrm{X}_{2}$ ermittelt:

$$
\begin{aligned}
& x_{1}=0,158781 \mathrm{t} \\
& x_{2}=-25,352571 \mathrm{~m}^{2} \mathrm{t}
\end{aligned}
$$

\section{Tabelle 2}

$E \delta_{i k}=\int_{0}^{\ell} \frac{M_{x i} M_{x k}}{I_{x x}} d z+\int_{0}^{\ell} \frac{M_{\omega i} M_{\omega k}}{I_{\omega \omega}} d z$

$\mathrm{E} \delta_{11}=11,698692+1,436039=13,134731$

$\mathrm{E} \delta_{12}=0 \quad-0,084615=-0,084615$

$\mathrm{E} \delta_{22}=0+0,014876=0,014876$

$\mathrm{E} \delta_{10}=0 \quad-4,230762=-4,230762$

$E \delta_{20}=0+0,390573=0,390573$

Durch Superposition werden die endgültigen Schnittkräfte erhalten (Bild 13), die mit denjenigen in Bild 12 übereinstimmen.

Wenn die horizontale Wirkungsebene des Biegemomentes $M_{x}$ durch die Punkte 1 und 4 gelegt wird, so entstehen im ganzen Stab Wolbmomente (Bild 12). Die überzähligen Grössen lauten in diesem Fall:

$$
\begin{aligned}
& x_{1}=0,158781 \mathrm{t} \\
& x_{2}=-16,206775 \mathrm{~m}^{2} \mathrm{t}
\end{aligned}
$$

Mit dem Wölbmomentenanteil von $\mathrm{X}_{1}$ an der Einspannstelle

$$
-57,6 \cdot 0,158781=-9,145796 \mathrm{~m}^{2} t
$$

beträgt das resultierende Wölbmoment:

$$
\mathbb{M}_{\omega}=-25,352571 \mathrm{~m}^{2} \mathrm{t}
$$




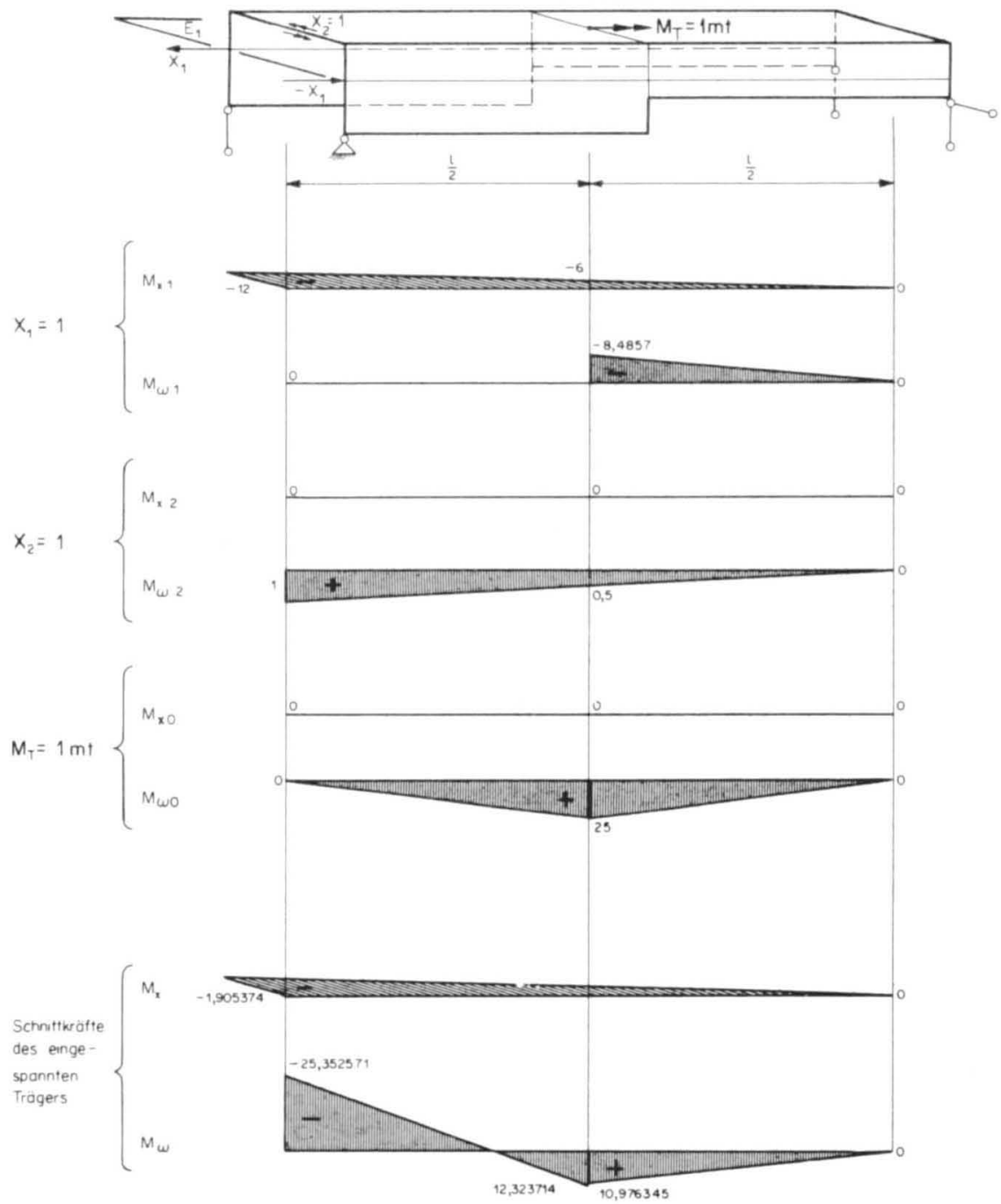

Bild 13 Schnittkräfte des Grundsystems und des eingespannten Trägers bei zwei Ueberhähligen (mit Ausnutizung der Symmetrie) 
Das Biegemoment $\mathrm{M}_{\mathrm{x}}$ an der Einspannstelle ist

$$
\begin{aligned}
& M_{x}=-12 \cdot 0,158781 \\
& M_{x}=-1,905372 \mathrm{mt}
\end{aligned}
$$

\subsection{Durchlaufender Stab}

Bei der Torsionsberechnung des durchlaufenden Stabes wird eine Reihe von einfachgelagerten Trägern als Grundsystem gewählt. Dabei werden zwei Nachbarträger in einem gemeinsamen Querschnittspunkt zusammengehalten. Die Verträglichkeitsbedingungen müssen bei unsymmetrischen, variablen Querschnitten in drei weiteren gemeinsamen Querschnittspunkten formuliert werden, so dass pro Zwischenstiutze drei Ueberzählige eingefuhrt werden. Da der Einfluss dieser Ueberzähligen sich nur auf die beiden Nachbarstäbe erstreckt, enthält das Gleichungssystem nur Glieder im Bereich der Diagonalen. Bei einfachsymmetrischen Querschnitten reduziert sich die Zahl der Ueberhähligen auf zwei: $M_{x}$ und $M_{\omega}$. 


\section{VERGLEICH MIT DER FALTWERKTHEORIE}

Im Falle verschwindender St. Venantscher Torsionssteifigkeit können aus ebenen Scheiben bestehende, dünwandige stäbe auch als Faltwerke berechnet werden. Im allgemeinen ist die Membrantheorie der prismatischen Faltwerke (Dreischübe- bzw. Dreispannungsgleichungen [11] ) für Faltwerke, deren Scheibenquerschnitte in Längsrichtung veränderlich sind, nicht anwendbar. Im folgenden wird gezeigt, dass die Dreischübegleichungen für den Kragträger und den Stab mit Gabellagerung direkt angeschrieben werden können. Danach werden diese zwei Systeme sowohl als Faltwerk als auch als dünnwandiger Stab nach Gl. (19) berechnet.

Damit die Resultate der Stab- und Faltwerktheorie direkt miteinander verglichen werden können, wird als Beispiel ein aus nur drei Scheiben bestehender Stab gewählt. Bei mehr als drei Scheiben unterscheiden sich die beiden Lösungen um einen Eigenspannungszustand, welcher den Einfluss der Querschnittsverformung angibt (siehe [2]).

Aus ebenen Tragflächen bestehende räumliche Tragwerke hat schon H. SCHWYZER [12] untersucht. Er zeigt, dass bei "statisch bestimmter Bauart" dieser Tragwerke die Auflagerkräfte und die Kantenkräfte mit Hilfe der Gleichgewichtsbedingungen allein bestimmt werden können. Mit diesen Kantenkräften und den auf die Scheibe wirkenden äusseren Kräften berechnete er die Randspannungen und setzte die zusammengehörigen Werte einander gleich. Aus diesen Gleichungen wurden die unbekannten Teilkantenkräfte und somit die Verteilung der Kantenkraft längs der Kante ermittelt.

\subsection{Berechnung als Faltwerk}

In Bild 14 ist ein Kragträger dargestellt, der aus drei Scheiben besteht. Es wird vorausgesetzt, dass jede Scheibe nur in ihrer Ebene belastet wird. 


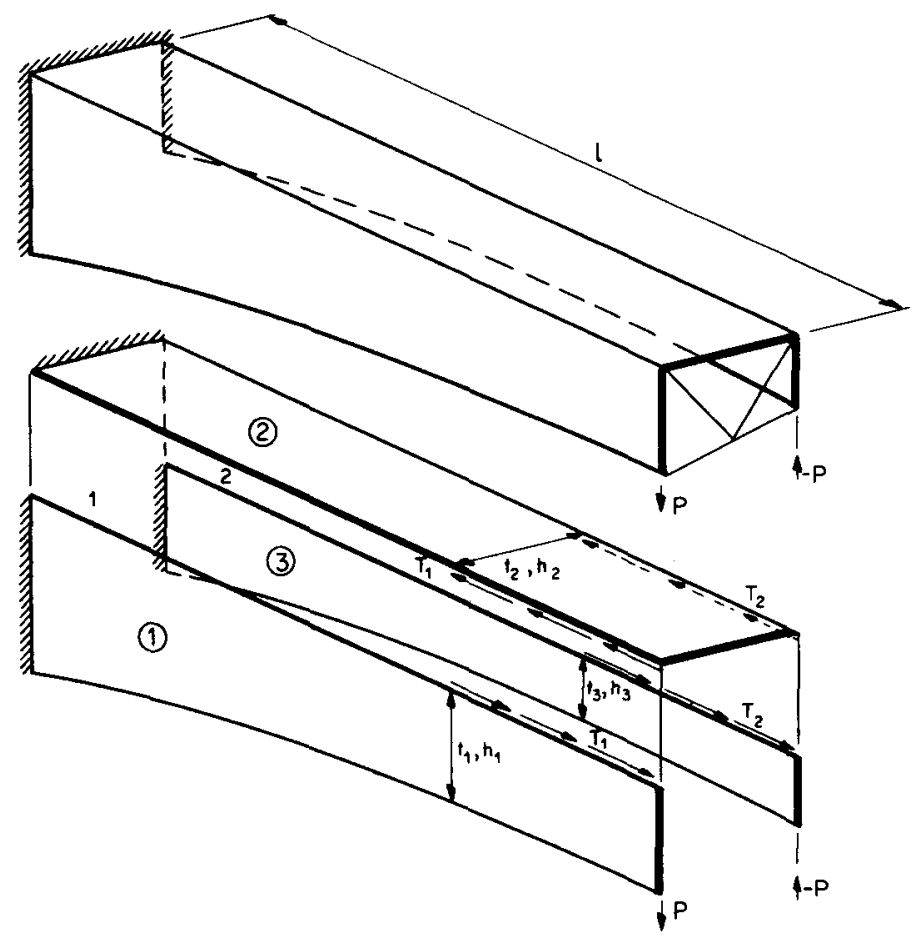

Bild 14 Berechnung des Kragträgers als Faltwerk

Zur Berechnung wird das Tragwerk längs den Kanten aufgeschnitten und die Verträglichkeitsbedingung, welche die Gleichheit der Dehnungen in den Randfasern der beiden Scheiben verlangt, in jedem Kantenpunkt eines Querschnittes formuliert. Das Grundsystem besteht somit aus drei statisch bestimmt gelagerten Balken, d.h. den drei Kragbalkem. Mit dem Kantenschub $\mathrm{T}_{\boldsymbol{k}}$ vom freien Ende bis zum betrachteten Schnitt kann die Randspannung $\sigma_{k i}$ der Scheibe $i$ in der Kante $k$ angeschrieben werden, wobei $t_{i}$ die konstante Scheibenstärke und $h_{i}$ die Scheibenhöhe im betrachteten Querschnitt sind. Es ist zu beachten, dass die Schubflüsse links vom Schnitt keinen Einfluss auf die Spannungen haben. 
Mit $F_{i}=t_{i} h_{i}$ und $W_{i}=t_{i} h_{i}^{2} / 6$ lauten die Randspannungen in der Kante I:

$$
\begin{aligned}
& \sigma_{11}=\sigma_{11}^{0}+\frac{T_{1}}{F_{1}}+\frac{T_{1} h_{1}}{2 W_{1}} \\
& \sigma_{12}=\sigma_{12}^{0}-\frac{T_{1}}{F_{2}}-\frac{T_{2}}{F_{2}}-\frac{T_{1} h_{2}}{2 W_{2}}+\frac{T_{2} h_{2}}{2 W_{2}}
\end{aligned}
$$

Dabei bedeuten $\sigma_{11}^{0}$ und $\sigma_{12}^{0}$ die Randspannungen am Grundsystem. Analog gilt fur Kante 2:

$$
\begin{aligned}
& \sigma_{23}=\sigma_{23}^{0}+\frac{T_{2}}{F_{3}}+\frac{T_{2} h_{3}}{2 W_{3}} \\
& \sigma_{22}=\sigma_{22}^{0}-\frac{T_{2}}{F_{2}}-\frac{T_{1}}{F_{2}}-\frac{T_{2} h_{2}}{2 W_{2}}+\frac{T_{1} h_{2}}{2 W_{2}}
\end{aligned}
$$

Die Verträglichkeitsbedingungen für die beiden Kanten sind

$$
\sigma_{11}=\sigma_{12} \quad \text { und } \quad \sigma_{23}=\sigma_{22}
$$

Somit entsteht das Gleichungssystem (30), das die unbekannten Schubfluisse $\mathrm{T}_{\mathrm{k}}$ liefert.

$$
\begin{array}{ll}
\mathrm{T}_{1}\left(\frac{1}{\mathrm{~F}_{1}}+\frac{1}{\mathrm{~F}_{2}}+\frac{\mathrm{h}_{1}}{2 \mathrm{~W}_{1}}+\frac{\mathrm{h}_{2}}{2 \mathrm{~W}_{2}}\right)+\mathrm{T}_{2}\left(\frac{1}{\mathrm{~F}_{2}}-\frac{\mathrm{h}_{2}}{2 \mathrm{~W}_{2}}\right) & +\sigma_{11}^{0}-\sigma_{12}^{0}=0 \\
\mathrm{~T}_{1}\left(\frac{1}{\bar{F}_{2}}-\frac{\mathrm{h}_{2}}{2 \mathrm{~W}_{2}}\right) & +\mathrm{T}_{2}\left(\frac{1}{\mathrm{~F}_{2}}+\frac{1}{\mathrm{~F}_{3}}+\frac{h_{2}}{2 W_{2}}+\frac{h_{3}}{2 W_{3}}\right)+\sigma_{23}^{0}-\sigma_{22}^{0}=0
\end{array}
$$

Mit $w_{i}=F_{i} h_{i} / 6, \Delta \sigma_{1}=\sigma_{11}^{0}-\sigma_{12}^{0}$ und $\Delta \sigma_{2}=\sigma_{23}^{0}-\sigma_{22}^{0}$ lautet das Gleichungssystem:

$$
\begin{array}{r}
\mathrm{T}_{1} \cdot 4\left(\frac{1}{\mathrm{~F}_{1}}+\frac{1}{\mathrm{~F}_{2}}\right)-\mathrm{T}_{2} \cdot 2 \cdot \frac{1}{\mathrm{~F}_{2}}=-\Delta \sigma_{1} \\
-\mathrm{T}_{1} \cdot 2 \cdot \frac{1}{\mathrm{~F}_{2}}+\mathrm{T}_{2} \cdot 4\left(\frac{1}{\mathrm{~F}_{2}}+\frac{1}{\mathrm{~F}_{3}}\right)=-\Delta \sigma_{2}
\end{array}
$$

Fiir diesen einfachen Fall können die geschlossenen Lösungen angegeben werden 


$$
\begin{aligned}
& \mathrm{T}_{1}=\frac{-\Delta \sigma_{1} \cdot 4\left(\frac{1}{\mathrm{~F}_{2}}+\frac{1}{\mathrm{~F}_{3}}\right)-\Delta \sigma_{2} \cdot \frac{2}{\mathrm{~F}_{2}}}{16\left(\frac{1}{\mathrm{~F}_{1} \mathrm{~F}_{2}}+\frac{1}{\mathrm{~F}_{1} \mathrm{~F}_{3}}+\frac{1}{\mathrm{~F}_{2}^{2}}+\frac{1}{\mathrm{~F}_{2} \mathrm{~F}_{3}}\right)-\frac{4}{\mathrm{~F}_{2}^{2}}} \\
& \mathrm{~T}_{2}=\frac{-\Delta \sigma_{2} \cdot 4\left(\frac{1}{\mathrm{~F}_{4}}+\frac{1}{\mathrm{~F}_{2}}\right)-\Delta \sigma_{1} \cdot \frac{2}{\mathrm{~F}_{2}}}{16\left(\frac{1}{\mathrm{~F}_{1} \mathrm{~F}_{2}}+\frac{1}{\mathrm{~F}_{1} \mathrm{~F}_{3}}+\frac{1}{\mathrm{~F}_{2}{ }^{2}}+\frac{1}{\mathrm{~F}_{2} \mathrm{~F}_{3}}\right)-\frac{4}{\mathrm{~F}_{2}^{2}}}
\end{aligned}
$$

a)

b)
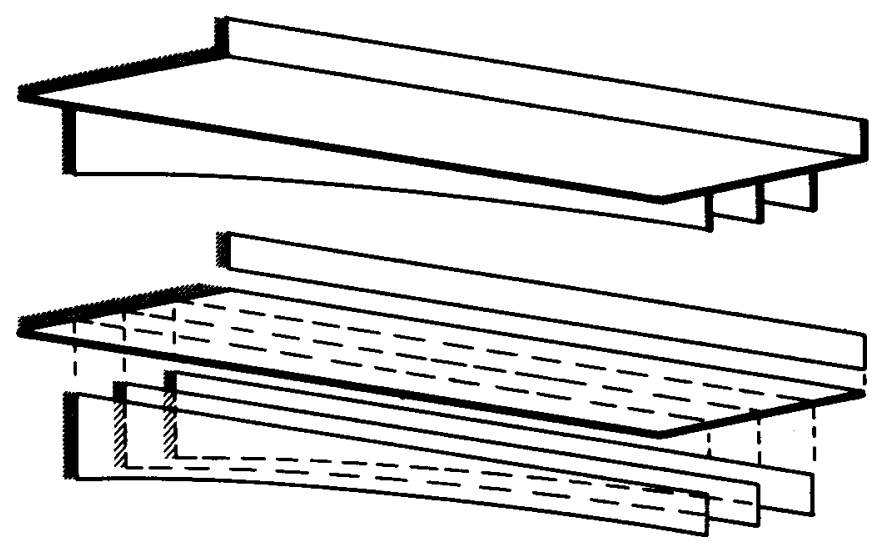

c)

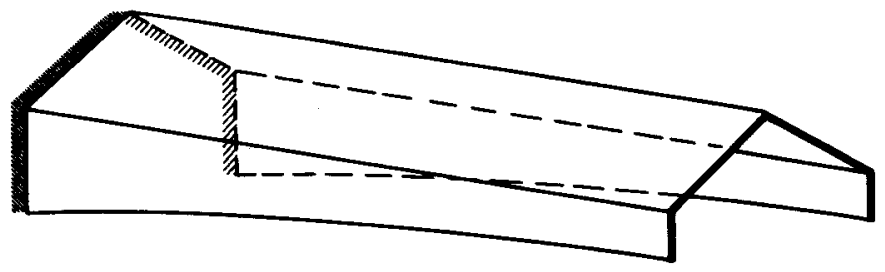

d)

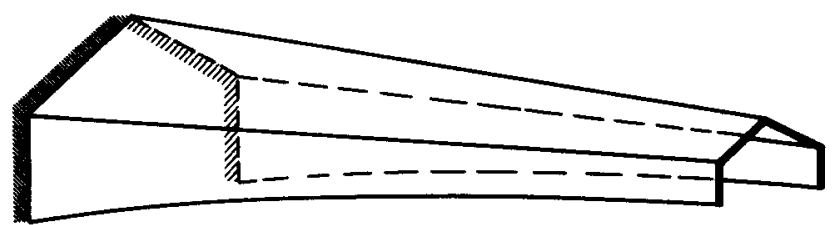

Bild 15 Faltwerke mit mehr als drei Scheiben 
Aus den Gleichungen (32) ist ersichtlich, dass der Kantenschub $\mathrm{T}_{\mathrm{k}}$ nur von der Geometrie des betrachteten Querschnittes und den Randspannungen des Grundsystems abhängt. Aendert sich der Trägerquerschnitt sprunghaft, so entstehen links und rechts der Sprungstelle verschieden grosse $T_{k}$. Bei unstetiger Querschnittsänderung ist somit der Verlauf von $\mathrm{T}_{\mathrm{k}}$ auch unstetig; d.h. an den Sprungstellen treten konzentrierte Schubkräfte auf. Das gleiche Problem stellt sich auch bei Verbundträgern und bei Stahlträgern mit Lamellenverstärkungen. Die tatsächlichen Verhältnisse an der Sprungstelle werden im Anhang besprochen.

Analog können auch die mehrstegigen Plattenbalken berechnet werden (Bild 15a,b). In diesem Fall entstehen so viele Gleichungen, wie Stege bzw. Kanten vorhanden sind. Das in Bild 15c dargestellte System liefert Dreischübegleichungen, welche näherungsweise auch für den Träger in Bild 15d guiltig sind (siehe Kapitel 7). Durch die Anordnung von genügend vielen Querschotten können die Querschnittsverformungen eliminiert werden. In diesem Fall entsteht im Träger der Spannungszustand des dünnwandigen stabes mit nicht deformierbarem Querschnitt. Auch der Einfluss elastischer Querträger oder Querrahmen kann einfach erfasst werden.

Als nächstes Beispiel für eine Lagerung, welche die einfache Ermittlung der Spannungen im Faltwerk erlaubt, soll der in Bild 16a gezeigte Dreischeibenträger betrachtet werden. Wiederum werden für die Berechnung die Scheiben voneinander getrennt. Damit das räumłiche System nicht instabil wird, werden sie in einem Punkt, z.B. am Stabanfang, miteinander gelenkig verbunden. Dieses Grundsystem besteht also aus arei in ihren Ebenen statisch bestimmt gelagerten Trägerm, d.h. drei einfachen Balken in verschiedenen Ebenen. Die Wirkung der Endscheiben soll durch die zwei horizontalen Pendelstäbe der Scheibe 2 veranschaulicht werden (Bild 16b).

Die Randspannungen für die Kante I lauten:

$$
\begin{aligned}
& \sigma_{11}=\sigma_{11}^{0}+T_{1}\left(\frac{l}{\mathrm{~F}_{1}}+\frac{h_{1}}{2 W_{1}}\right) \\
& \sigma_{12}=\sigma_{12}^{0}-T_{1}\left(\frac{1}{F_{2}}+\frac{h_{2}}{2 W_{2}}\right)-T_{2}\left(\frac{1}{F_{2}}-\frac{h_{2}}{2 W_{2}}\right)
\end{aligned}
$$




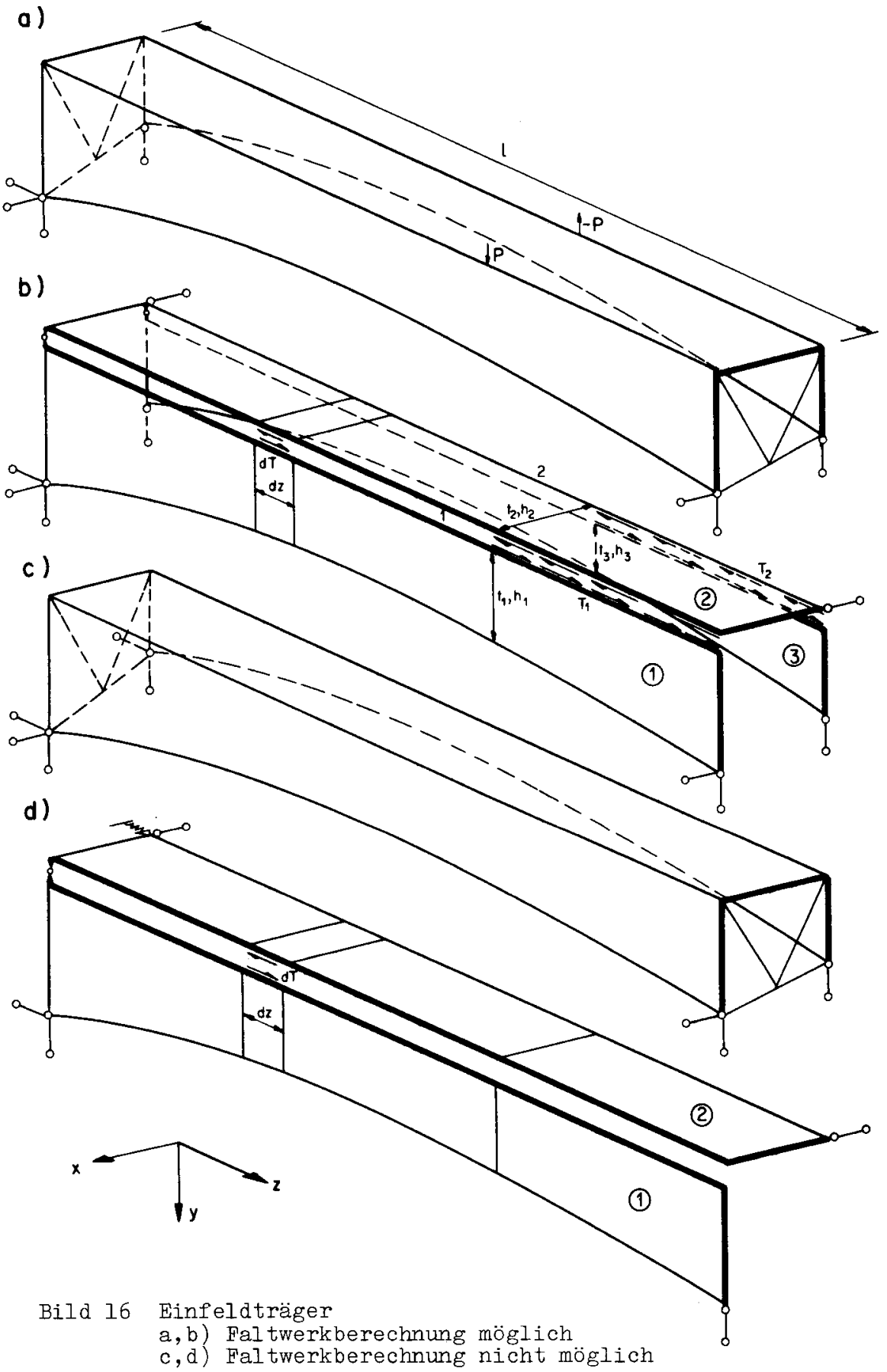


Ein Vergleich mit dem oben behandelten Kragträger zeigt, dass die Gleichungen (28) und (33) identisch sind. Somit haben die Gleichungen (3I) und (32) auch für diesen Träger Gültigkeit.

Daraus kann geschlossen werden, dass die Faltwerkberechnung immer dann möglich wird, wenn die einzelnen Scheiben nach der Trennung eine statisch bestimmte Lagerung aufweisen. (Statisch bestimmte Bauart).

Wird der Träger 3 am linken Ende festgehalten (Bild 16c), wie es im Brickenbau üblich ist, dann sind die Scheiben nicht mehr statisch bestimmt gelagert (Bild 16d). In diesem Fall sind die Gleichungen (33) nicht mehr guiltig, weil auch die Schubkräfte dT links vom betrachteten Schnitt die Spannungen beeinflussen.

Durch die Einführung der neuen Lagerkraft in Stablängsrichtung als überzählige Grösse kann dàs Problem gelöst werden. Das System wäre auch statisch bestimmt, wenn man den Pendelstab parallel zur $\mathbf{x}$-Achse am rechten Ende entfernen wirde.

Analog werden Faltwerke mit mehr als drei Scheiben behandelt, dabei soll an den Einfluss der Querschnittsverformung erinnert werden: In solchen Faltwerken unterscheidet sich der Spannungszustand von demjenigen des dinnwandigen Stabes.

\subsection{Beispiele}

Anhand zweier Beispiele soll gezeigt werden, dass beim Dreischeibenquerschnitt die Resultate der Faltwerkberechnung und der Differentialgleichung (19) übereinstimmen, wenn die St. Venantsche Torsionssteifigkeit vernachlässigbar ist.

Der Kragträger in Bild 17a soll am. freien Ende durch ein Torsionsmoment von $\mathrm{M}_{\mathrm{T}}=100 \mathrm{cmkg}$ belastet werden. Die Abressungen sind aus dieser Abbildung ersichtlich. Zunächst wird der Träger als Faltwerk betrachtet (Bild 17b). Das Torsionsmoment wird durch das 
a)

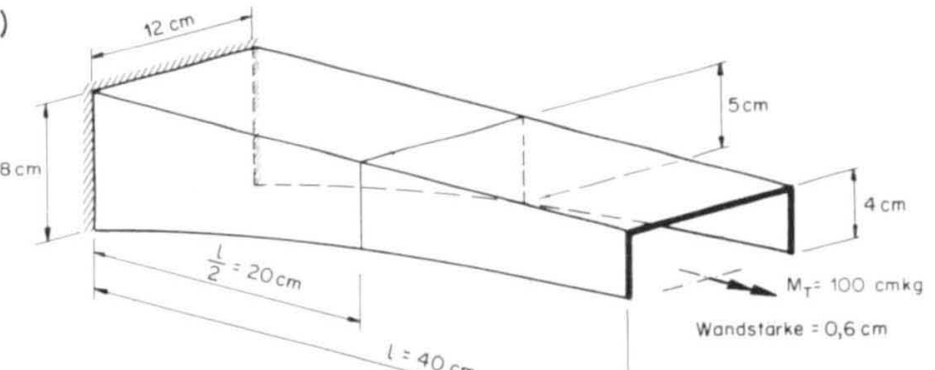

b)

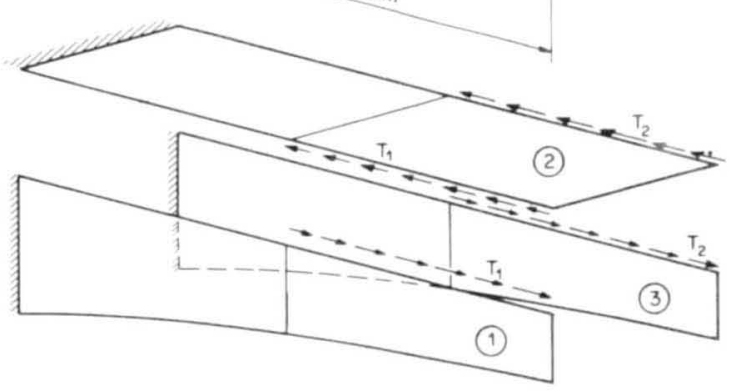

c)
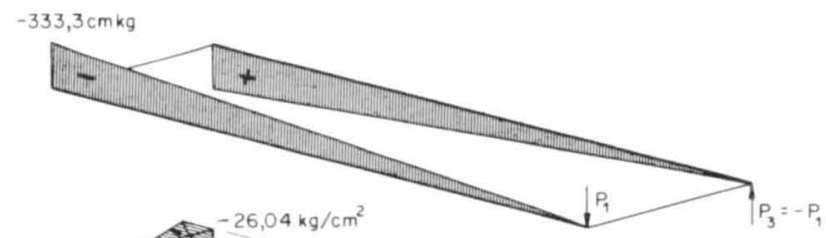

e)

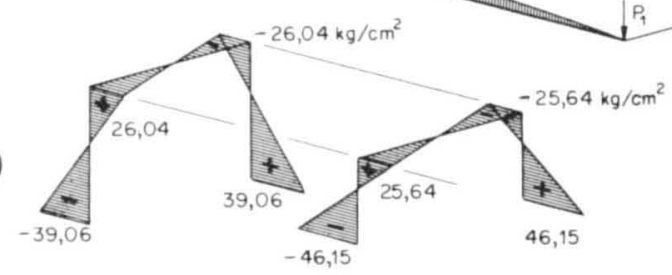

d)

$$
\begin{aligned}
& \begin{aligned}
F_{1}=F_{3} & =4,8 \mathrm{~cm}^{2} \\
F_{2} & =7,2 \mathrm{~cm}^{2}
\end{aligned} \\
& \begin{aligned}
F_{1}=F_{3} & =3,0 \mathrm{~cm}^{2} \\
F_{2} & =7,2 \mathrm{~cm}^{2}
\end{aligned} \\
& W_{1}=W_{3}=6,4 \mathrm{~cm}^{3} \\
& W_{2}=14,4 \mathrm{~cm}^{3} \\
& \begin{aligned}
W_{1}=W_{3} & =2,5 \mathrm{~cm}^{3} \\
W_{2} & =14,4 \mathrm{~cm}^{3}
\end{aligned}
\end{aligned}
$$

Bild 17 Kragträger. Berechnung als Faltwerk
a) System und Belastung
b) Grundsystem
c) Nomente am Grundsystem
d) Spannungen in Stabmitte
e) Spannungen am Einspannquerschnitt 
Kräftepaar $P_{1}, P_{3}$ ersetzt.

$$
P_{1}=-P_{3}=\frac{100}{12}=8,33 \mathrm{~kg}
$$

Die Spannungen sollen in Stabmitte und an der Einspannstelle berechnet werden. Die Randspannungen am Grundsystem werden mit den Momenten des Bildes $17 \mathrm{c}$ und den Widerstandsmomenten, die in Bild $17 d$ angeschrieben sind, ermittelt.

Mit den Randspannungen des Grundsystems in der Kante 1

$$
\sigma_{11}^{0}=\frac{166,7}{2,5}=66,7 \mathrm{~kg} / \mathrm{cm} \text { und } \sigma_{12}^{0}=0
$$

wird mit Gleichung (32) der Kantenschub vom freien Ende bis zur Stabmitte gerechnet:

$$
\mathrm{T}_{1}=-30,77 \mathrm{~kg}
$$

Die Gleichungen (28) liefern die Randspannungen in der Kante 1. Wegen der Symmetrie des Trägers sind die Randspannungen der Kante 2 bis auf die Vorzeichen die gleichen (Bild 17d). Analog ist in der Einspannstelle:

$$
\begin{aligned}
& \sigma_{11}^{0}=\frac{333,3}{6,4}=52,1 \mathrm{~kg} / \mathrm{cm}, \quad \sigma_{12}^{0}=0 \\
& T_{1}=-31,25 \mathrm{~kg}
\end{aligned}
$$

Die Spannungsverteilung ist aus Bild $17 \mathrm{e}$ ersichtlich.

Der gleiche Kragträger soll auch mit der Differentialgleichung (19)

$$
\left(E I_{\omega \omega} \varphi^{\prime \prime}\right)^{\prime \prime}=\mathrm{m}_{\text {T }}
$$

als dïnnwandiger Stab berechnet werden. Dazu wird von der bereits erwähnten Analogie zwischen Biegung und Verdrehung Gebrauch gemacht. 
a)

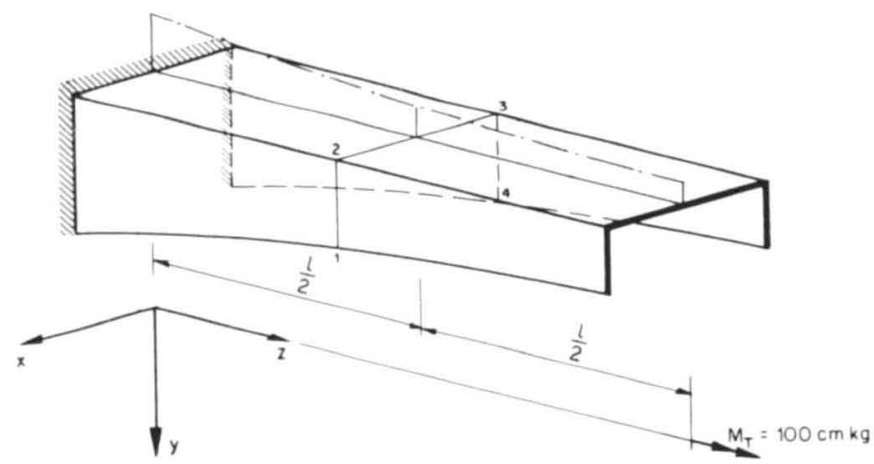

b)

d)
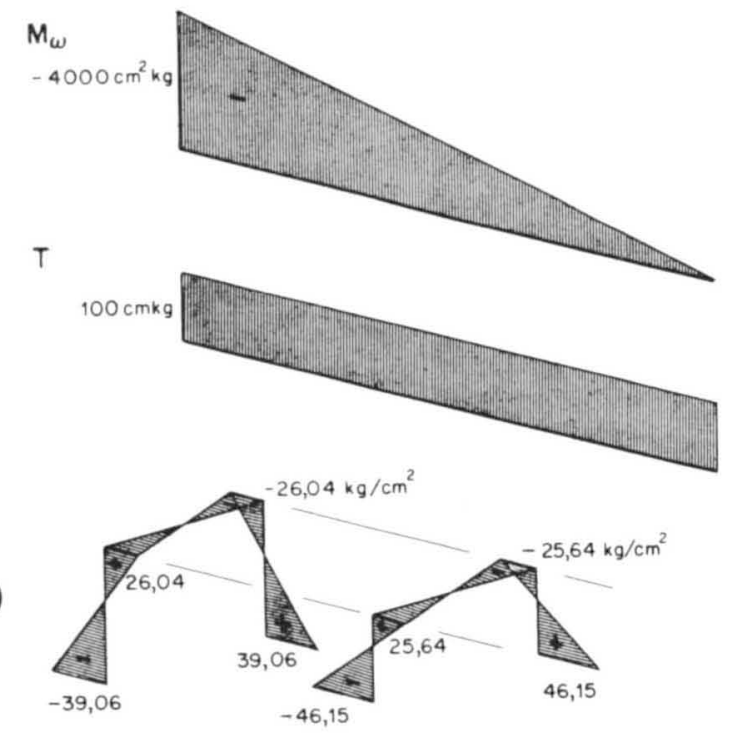

c)
$I_{\omega \omega}=2949,1200 \mathrm{~cm}^{6}$
$I_{\omega \omega}=835,7143 \mathrm{~cm}^{6}$
$\omega_{1}=28,8000 \mathrm{~cm}^{2}$
$\omega_{2}=-19,2000 \mathrm{~cm}^{2}$
$\omega_{1}=19,2857 \mathrm{~cm}^{2}$
$\omega_{2}=-10,7143 \mathrm{~cm}^{2}$

Bild 18 Kragträger. Berechnung als dinnwandiger Stab
a) System und Belastung
b) Schnittkräfte
c) Spannungen in Stabmitte
d) Spannungen im Einspannquerschnitt 
a)

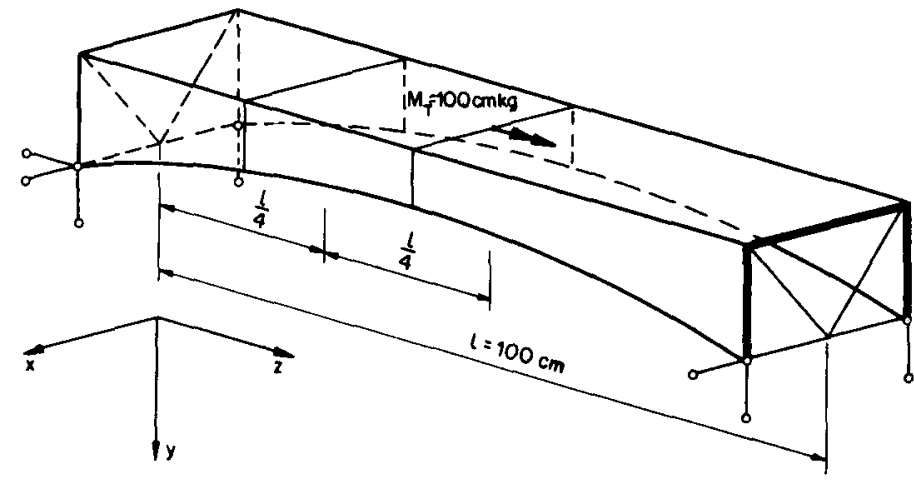

b)
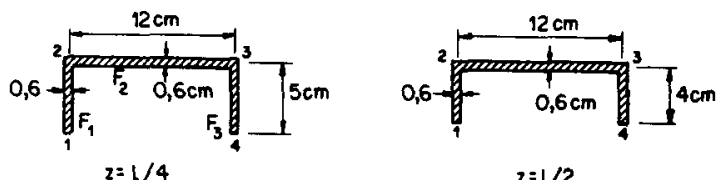

$$
\begin{aligned}
F_{1}=F_{3} & =3,0 \quad \mathrm{~cm}^{2} \\
F_{2} & =7,2 \quad \mathrm{~cm}^{2} \\
W_{1}=W_{3} & =2,5 \mathrm{~cm}^{3} \\
W_{2} & =14,4 \mathrm{~cm}^{3} \\
I_{\omega \omega} & =835,7143 \mathrm{~cm}^{6} \\
\omega_{1} & =19,2857 \mathrm{~cm}^{2} \\
\omega_{2} & =-10,7143 \mathrm{~cm}^{2}
\end{aligned}
$$

$$
\begin{aligned}
F_{1}=F_{3} & =2,4 \quad \mathrm{~cm}^{2} \\
F_{2} & =7,2 \quad \mathrm{~cm}^{2} \\
W_{1}=W_{3} & =1,6 \quad \mathrm{~cm}^{3} \\
W_{2} & =14,4 \quad \mathrm{~cm}^{3} \\
I_{\omega \omega} & =460,8000 \mathrm{~cm}^{6} \\
\omega_{1} & =16,0000 \mathrm{~cm}^{2} \\
\omega_{2} & =-8,0000 \mathrm{~cm}^{2}
\end{aligned}
$$

Bild 19 Träger mit Gabellagerung an beiden Enden unter Torsionsbelastung
a) System und Belastung
b) Querschnittswerte

In Bild 18 sind die Querschnittswerte sowie Wölb- und Torsionsmomente dargestellt. Mit den Wölbkoordinaten $\boldsymbol{\omega}_{1}$ und $\boldsymbol{\omega}_{2}$ der Eckpunkte des Querschnitts werden die Wölbnormalspannungen nach der Formel

$$
\sigma=\frac{M_{\omega}}{\bar{I}_{\omega \omega}} \omega
$$

bestinnt.

Der Spannungsverlauf in den betrachteten Querschnitten (Bild 18c,d) stimmt mit derjenigen in Bild 17d, e uberein. 
Als weiteres Beispiel werden die Spannungen in Stabmitte und im Viertelspunkt des Trägers mit Gabellagerung an beiden Enden ermittelt. Der Träger ist durch ein äusseres Drehmoment

$\mathrm{M}_{\mathrm{T}}=100 \mathrm{cmkg}$ belastet (Bild 19).

Mit den in Bild 19 angegebenen Abmessungen und Querschnittswerten wird die Berechnung als Faltwerk und als dünwandiger Stab durchgeführt. Die Spannungen und die für ihre Ermittlung erforderlichen Momente gehen aus Bild 20 hervor.

o )
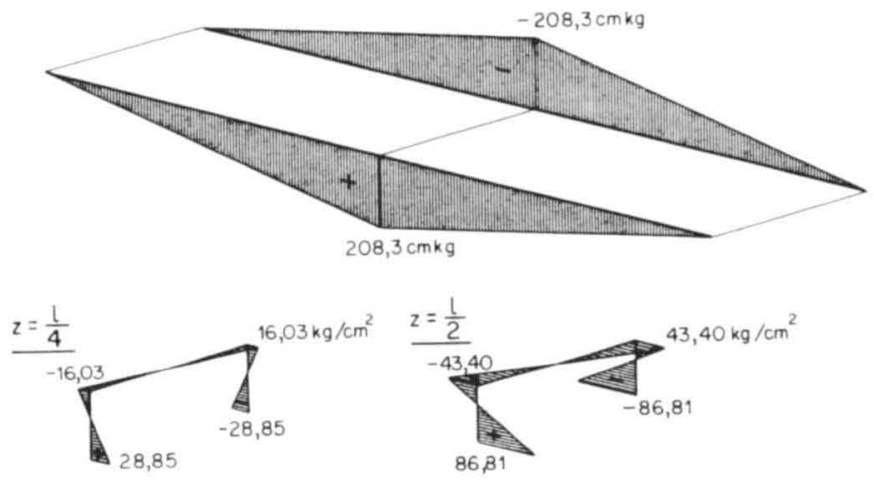

b )
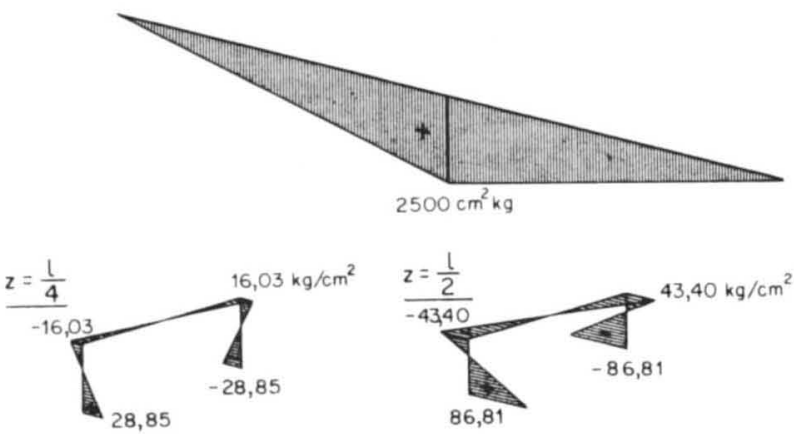

3ild 20 Träger mit Gabellagemung an beiden Enden unter Torsionsbelastung

a) Berechnung als Faltwerk

b) Berechnung als dinnwandiger stab 
6. BERUECKSICHTIGUNG DER ST. VENANTSCHEN TORSION

\subsection{Praktische Berechnung}

Das im Kapitel 3 besprochene Trägermodell gibt die Möglichkeit, den dünnwandigen Stab mit offenem Querschnitt unter Torsionsbelastung als ein statisch unbestimmtes Stabsystem zu betrachten und das Torsionsproblem mit den üblichen Methoden der Baustatik zu lösen, wenn es durch die Differentialgleichung (18) beschrieben werden kann. Deshalb liefert das Modell nur im Falle des Kragträgers und des Stabes mit Gabellagerung an beiden Enden sowie bei beliebig gelagerten prismatischen Stäben direkt die richtigen Resultate.

Mit dem Modell können folgende Randbedingungen erfasst werden:

- Verdrehung der Stabquerschnitte (Stab W: $\delta$, Stab $S: \varphi$ )

- Verwölbung der Stabquerschnitte (Stab W: $\delta^{\prime}$ )

Da unter Torsionsbelastung die Endquerschnitte eines Stabes mit variablem Querschnitt auch um die Achsen $x$ und $y$ gedreht werden (Kapitel 4), können bei der Berechnung mit dem Modell nicht alle Randbedingungen berücksichtigt werden. Deshalb liefert das Modell bei beliebiger Lagerung solcher Stäbe nur Näherungslösungen. Wird dieses System als statisch unbestimntes Grundsystem aufgefasst, und werden die nicht berücksichtigten Biegemomente $\mathbb{M}_{x}, \mathbb{M}_{y}$ und Normalkräfte $N$ als Ueberzählige eingeführt, so können die richtigen Schnittkräfte gewonnen werden. Meistens weichen die Schnittkräfte des statisch unbestimmten Grundsystems von denjenigen des tatsächlichen Systems nicht stark $a b$, deshalb stellen sie gute Näherungswerte dar. Die Grössenordnung des maximalen Fehlers kann jedoch einfach abgeschätzt werden, inden jeweils nur eine der Ueberzähligen berücksichtigt wird, weil die so ermittelten Werte grösser sind als die tatsächlichen.

Wird der Kragträger oder der Stab mit Gabellagerung an beiden Enden als statisch bestimmtes Grundsystem gewählt (innerlich statisch unbestimmt), so müssen auch die Wölbmomente als Ueberzäh- 
lige eingeführt werden. Es können auch einzelne Festhaltekräfte die Ueberzählige bilden, wie dies im Kapitel 4 gezeigt wurde. Die totale Einspannung kann mit vier Festhaltekräften erzielt werden.

Die Berechnung beliebig gelagerter Stäbe mit variablem Querschnitt mit Hilfe des Modells kann auch auf folgende Art erfolgen, die jedoch umständlicher ist. Die inneren Bindungen der beiden Teilstäbe werden gelöst, und die unbekannten Bindungskräfte werden als Ueberzählige eingefuhrt. Die Verdrehungen der Teilstäbe infolge der Ueberzähligen und der äusseren Belastung werden bei unveränderter äusserer Lagerung ermittelt. Dabei wird der Stab mit st. Venantscher Torsionssteifigkeit GK nach den bekannten Verfahren der Torsionstheorie, der ideal dünwandige Teilstab mit der Steifigkeit $\mathrm{EI}_{\boldsymbol{\omega} \boldsymbol{\omega}}$ nach der in Kapitel 4 angegebenen Methode berechnet. Die Verträglichkeitsbedingungen liefern die Unbekannten, mit denen die endgültigen Schnittkräfte ermittelt werden.

Die erste Berechnungsmethode eignet sich vor allem für die Anwendung von STRESS, indem möglichst viele Randbedingungen am statisch unbestimmten Grundsystem bericksichtigt werden. Die Einheitsbelastungen der Ueberzähligen werden als neue Lastfälle gerechnet. 


\subsection{Beispiele}

Als erstes Beispiel soll der in Bild 11 dargestellte Träger mit Bericksichtigung der st. Venantschen Torsionssteifigkeit berechnet werden. Mit den in Bild 13 angegebenen äusseren Ueberzähligen und 19 inneren Bindungen (Stablänge/20) wird die Berechnung nach beiden Methoden durchgeführt.

Die neuen Jeberzähligen lauten:

$$
\begin{aligned}
& x_{1}=0,128 t \\
& x_{2}=-21,7 \quad m^{2} t
\end{aligned}
$$

Die St. Venantsche Torsionssteifigkeit vermindert somit das Biegemoment $M_{X}$ um ca. $24 \%$ und das Wölbmoment $M_{\omega}$ um ca. $17 \%$.

Als zweites Beispiel wird der von Z. CYWIŃSKI [3] mit Differenzengleichungen behandelte Modellbalken nachgerechnet. Dazu wird der Stab in 20 Segmente mit konstantem Querschnitt unterteilt. Wegen der Symmetrie des Systems und der Belastung kann nur eine Stabhälfte betrachtet werden. Als statisch unbestimmtes Grundsystem wird der Träger mit starren Endblöcken gewählt und das Biegemoment $M_{x}=-X_{1}$ d als überzählige Grösse eingeführt (Bild 21 ). Die Resultate stimmen mit den in [3] angegebenen sehr gut überein.

Ein Grundsystem ohne die starren Endblöcke, bei dem neben $\mathrm{M}_{\mathrm{x}}$ auch das Wolbmoment $M$ an der Einspannstelle als Ueberzählige eingeführt wird, liefert die gleichen Resultate. Die Konvergenz ist jedoch besser, je weniger sich das Grundsystem vom Gegebenen unterscheidet. Solche kompliziertere Grundsysteme können mit STRESS sehr einfach behandelt werden. 


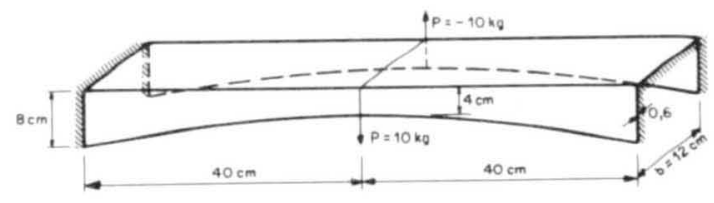

Grundsystem:

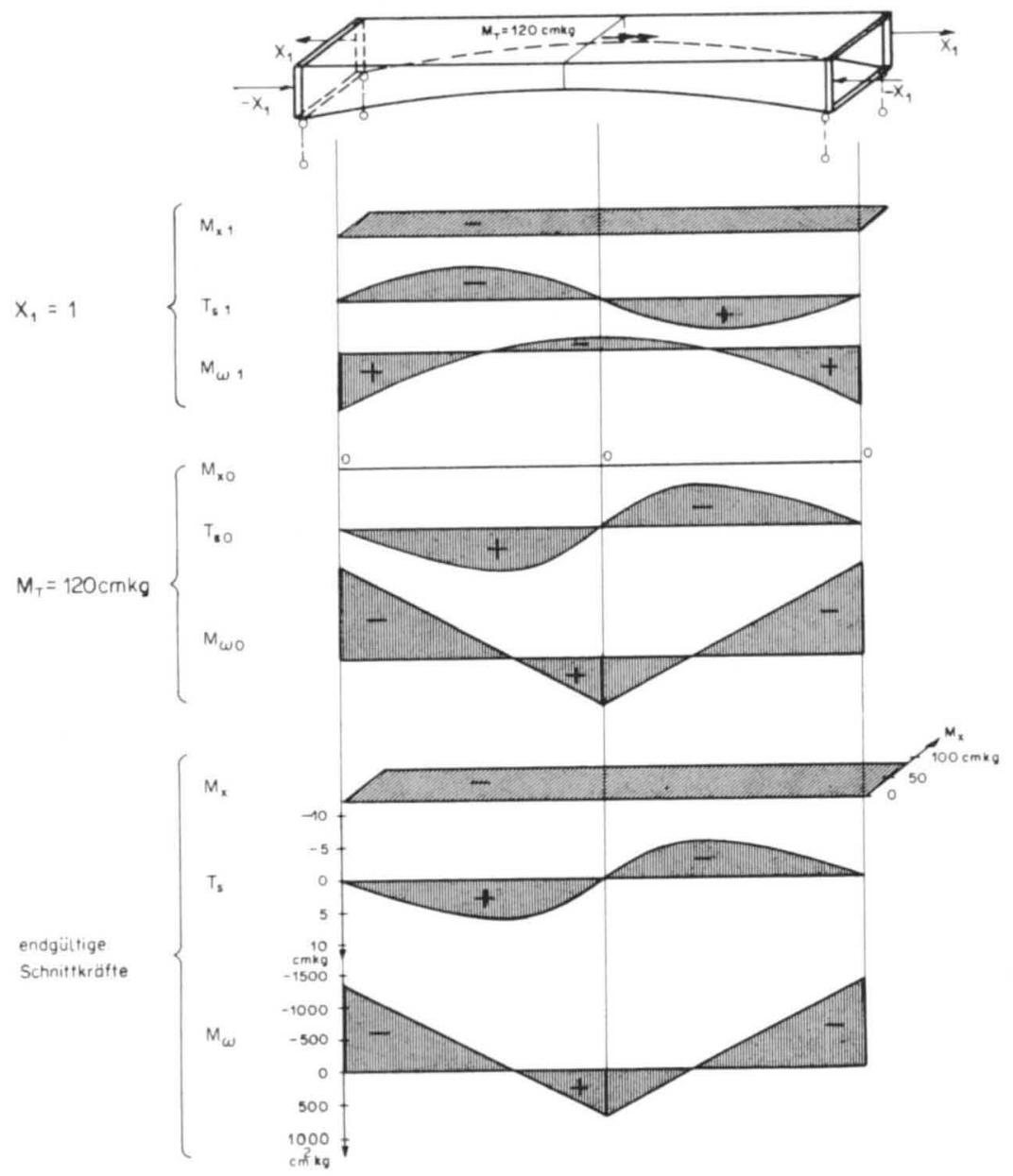

Bild 21 Berechnung des eingespanten Balkens unter Torsionsbelastung 
7. ALIGGMEINE VERAENDERLICHKEIT DES QUERSCHNITTS

Ist die Voraussetzung, dass die Mittelflächen der Wandelemente parallel zur Stablängsrichtung sein soll, nicht erfiillt, dann können die Spannungen und Dehnungen der Fasem nicht mehr senkrecht zur Querschnittsebene angenommen werden. In diesem Fall setzt sich das Torsionsmoment im Querschnitt nicht nur aus zwei Anteilen zusammen. Die Normalspannungen, die nicht parallel zur Stabachse verlaufen, liefern einen neuen Anteil.

Am Beispiel eines symmetrischen I-Trägers mit variabler Steghöhe, der nur aus zwei Flanschen besteht, soll das Verhalten solcher Stäbe gezeigt werden (Bild 22). Einfachheitshalber wird die St. Venantsche Torsionssteifigkeit vernachlässigt.

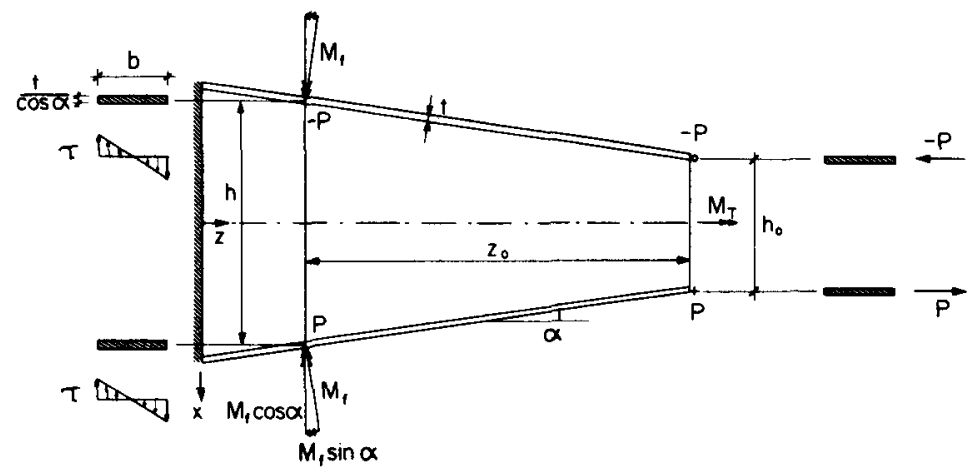

Bild 22 I-Träger mit variabler Steghöhe

Der Träger ist an einem Ende eingespannt und am freien thde durch ein Kräftepaar $\mathrm{M}_{\mathrm{T}}=\mathrm{Ph}_{\mathrm{O}}$ belastet. Die Zerlegung der Flanschbiegemomente $\mathbb{M}_{f}$ im betrachteten Stabquerschnitt zeigt, dass diese einen Anteil $2 \mathrm{M}_{\mathrm{f}} \sin \alpha$ liefern, der dern äusseren Torsionsmoment entgegenwirkt. 
Das resultierende Torsionsmoment im Querschnitt ist

$$
\begin{aligned}
& \mathrm{T}=\mathrm{Ph}-2 \mathrm{M}_{f} \sin \alpha=P\left(\mathrm{~h}_{0}+2 \mathrm{z}_{0} \operatorname{tg} \alpha\right)-2 \frac{\mathrm{Pz} \mathrm{z}_{0}}{\cos \alpha} \sin \alpha \\
& \mathrm{T}=\mathrm{Ph}_{0}
\end{aligned}
$$

Die Zerlegung der Hauptspannungen parallel und senkrecht zur Querschnittsebene ergibt Normal- und Schubspannungen. Die Randspannung in $z$-Richtung lautet

$$
\theta=\frac{M_{f}}{I_{f}} \frac{b}{2} \cos ^{2} \alpha=\frac{P z_{o} b}{2 I_{f}} \cos \alpha
$$

wobei $I_{f}=t^{3} / 12$ ist. Die Schubspannungen in $x$-Richtung sind $\tau=\sigma \operatorname{tg} \alpha$. Ihr Verlauf geht aus Bild 22 hervor. Sie bilden die Komponente $M_{f} \sin \alpha$ des Flanschbiegemomentes.

Die Berechnung nach Kapitel 4 ergibt kleinere Randspannungen. Mit $M_{\omega}=M_{0} z_{0}=P h_{0} z_{0}, I_{\omega \omega}=h^{2} I_{f} / 2 \cos \alpha$ und $\omega=b h / 4$ lautet die Randspannung

$$
\begin{aligned}
& \sigma=\frac{M_{\omega}}{I_{\omega \omega}} \omega=\frac{P h_{0} z_{0}}{h^{2} I_{f}} \frac{b h}{4} 2 \cos \alpha \\
& \sigma=\frac{P z_{0} b}{2 I_{f}} \frac{h_{0}}{h} \cos \alpha
\end{aligned}
$$

Bei schwacher Veränderlichkeit wes Querschnittes $\left(h_{0} / h \approx 1\right)$ liefert diese Berechnung gute Näherungslösungen.

Bei Trägern mit beliebig aber schwach veränderlichen Querschnitten, die aus Scheiben zusammengesetzt sind, kann das Tragverhalten mit der Faltwerktheorie besser erfasst werden. Bei diesen Stäben ist die z-Richtung, d.h. die Stablängsrichtung, nicht mehr definiert wie beim ursprünglich prismatischen Träger nach Voraussetzung sechs. In diesem Fall wird eine z-Richtung angenommen. Da es sich um schlanke Stäbe handelt, ist diese Annahme gerechtfertigt. Die Endscheiben werden senkrecht zur z-Achse angeordnet. 
Am Beispiel eines Faltwerks mit drei Scheiben werden die Randspannungen am Grundsystem angeschrieben. Einfachheitshalber wird die obere Scheibe parallel zur xz-Ebene gelegt, so dass nur die Ränder dieser Scheibe mit der z-Achse die Winkel $\alpha_{1}$ und $\alpha_{2}$ bilden (Bild 23).
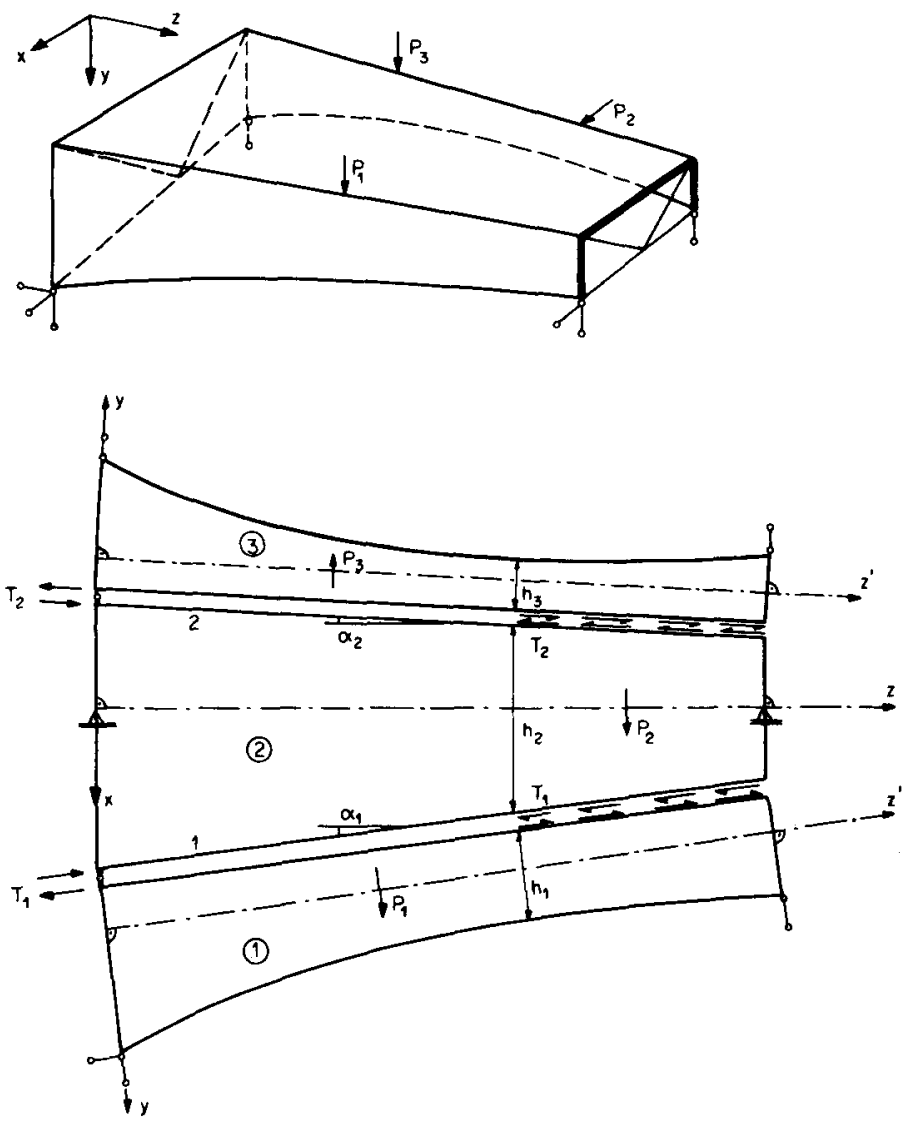

Bild 23 Faltwerk mit zusammenlaufenden Kanten 
Die Lagerung ist so gewählt, dass das Grundsystem statisch bestimmt wird. Die Randspannungen der Scheiben in der Kante 1 sind

$$
\begin{aligned}
& \sigma_{11}=\sigma_{11}^{0}+\mathbb{T}_{1}\left(\frac{1}{\mathrm{~F}_{1}}+\frac{h_{1}}{2 W_{1}}\right) \\
& \sigma_{12}=\sigma_{12}^{0}-\mathbb{T}_{1} \cos \alpha_{1}\left(\frac{1}{\mathrm{~F}_{2}}+\frac{h_{2}}{2 W_{2}}\right)-T_{2} \cos \alpha_{2}\left(\frac{1}{\mathrm{~F}_{2}}-\frac{h_{2}}{2 W_{2}}\right)
\end{aligned}
$$

Für $\alpha_{1}=\alpha_{2}=0$ gehen die Gleichungen (37) in Gleichungen (33) über. Analog Kapitel 5 werden die Schubflüsse $T_{k}$ und die endgültigen Randspannungen ermittelt. 


\section{ZUSAMMENFASSUNG}

In dieser Arbeit werden dïnnwandige Stäbe mit offenem, variablem Querschnitt unter Torsionsbelastung untersucht. Die Querschnittsveränderlichkeit der betrachteten stäbe entsteht dadurch, dass diese aus einem prismatischen Körper beliebig herausgeschnitten werden. Anhand der bekannten Differentialgleichungen wird gezeigt, dass bei lagerungsarten, die eine zwängungsfreie Verformung des Stabes erlauben, das Torsionsproblem durch eine einzige Differentialgleichung beschrieben werden kann. Für die lösung dieser linearen Differentialgleichung vierter Ordnung mit variablen Koeffizienten wird ein Modell zu Hilfe genommen, das die Benützung von STRESS und ähnlichen Rechenprógrammen zur Lösung des Torsionsproblems erlaubt.

Am zwängungsfrei gelagerten Träger, der für die Berechnung beliebig gelagerter Stäbe als Grundsystem dient, wird das unterschiedliche Torsionsverhalten von prismatischen stäben und solchen mit variablen Querschnitten erklärt, wobei die St. Venantsche Torsionssteifigkeit zunächst vernachlässigt wird. Diese Grundsysteme, d.h. der Kragträger und der Stab mit Gabellagerung, werden auch nach der Faltwerktheorie berechnet. An Stäben, die aus drei Scheiben bestehen, wird die Gleichheit der Resultate der Stab- und Faltwerktheorie gezeigt. Bei mehr als drei Scheiben können die Iösungen nicht direkt verglichen werden, weil sie sich um einen Eigenspannungszustand unterscheiden, der den Einfluss der Querschnittsverformung angibt.

Die Berücksichtigung der St. Venantschen Torsion erfolgt mit den Modell. Für den Kragträger und den Stab mit Gabellagerung an den Fnden liefert das Modell stets die richtigen Iösungen. Auch die Einspannung gegen Verwölbung (starre Enßblöcke) kann richtig erfasst werden. Bei beliebiger Lagerung werden Nomalkräfte und Biegemomente als Ueberzählige eingeführt. 
ANHAN G

\section{Einfiuss der verärderlichen Scheibenhöhe}

Laut Voraussetzung sieben werden die Spannungen und Dehnungen senkrecht zur Querschnittsfläche angenommen. Anhand einer Scheibe mit variabler Höhe soll der Gültigkeitsbereich dieser Voraussetzung abgeschätzt werden.

Die genaue Spannungsverteil.ung in keilförmigen Scheiben ist für verschiedene Belastungsfälle schon bekannt. Die in Bild $24 \mathrm{zu}-$ sammengestellten Fälle sind aus [13] entnommen worden.

Jede beliebige Belastung kann auf die Spitze des Keils reduziert und durch diese drei Komponenten ersetzt werden. In Bild 25 sind die Quotienten $\sigma_{r} / \sigma_{r}^{\circ}$ der Randspannungen einer keilförmigen Scheibe für drei typische Belastungen in Funktion des Winkels $2 \alpha$ dargestellt. Dabei ist $\sigma_{r}$ der exakte Wert der Randspannung am oberen Rand, während $\sigma_{r}^{\circ}$ am prismatischen stab mit der Höhe $h$ ermittelt wurde.

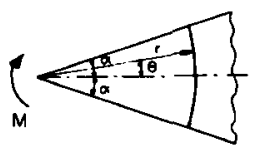

$\sigma_{r}=-\frac{2 M \sin 2 \theta}{(\sin 2 \alpha-2 \alpha \cos 2 \alpha) r^{2}}$

$\sigma_{\theta}=0$

$\tau_{r \theta}=\frac{M(\cos 2 \theta-\cos 2 \alpha)}{(\sin 2 \alpha-2 \alpha \cos 2 \alpha) r^{2}}$

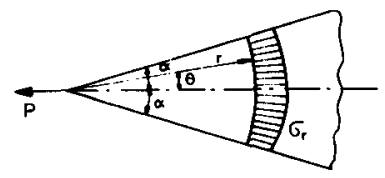

$\sigma_{r}=\frac{2 P}{2 \alpha+\sin 2 \alpha} \frac{\cos \theta}{r}$

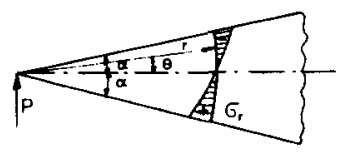

$\sigma_{r}=-\frac{2 \rho}{2 \alpha-\sin 2 \alpha} \frac{\sin \theta}{r}$

Bild 24 Spannungsverlauf in keilförmigen Scheiben (Scheibenstärke $=1$ ) 


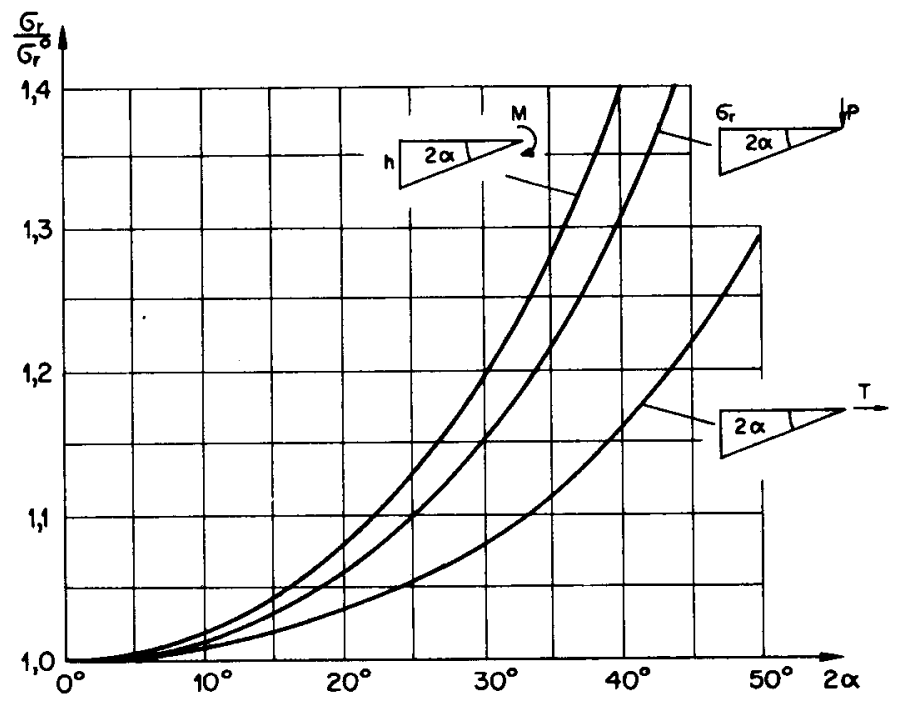

Bild 25 Randspannungen einer keilförmigen Scheibe für drei typische Belastungen. 


\section{Konzentrierte Schubkräfte bei Querschnittssprüngen}

Aus den Gleichungen (32) in Kapitel 5 folgt, dass bei plötzlicher Aenderung des Stabquerschnittes konzentrierte Kantenschubkräfte an der Sprungstelle auftreten. Anhand eines einfachen Beispiels

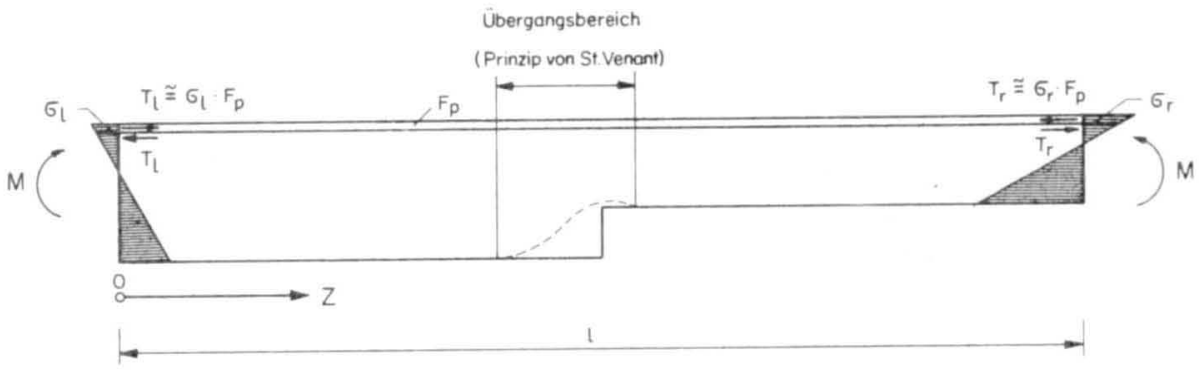

6

$\sigma=\sigma(z) \quad \sigma_{r}$ $\sigma_{1}$

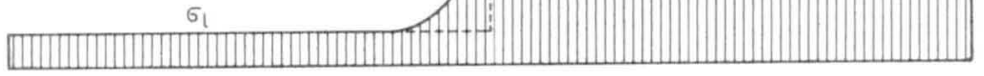

$\tau$

$\tau=0$

$\tau=\tau(z)$

:
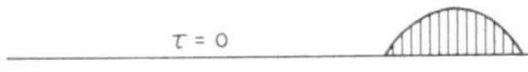

$\tau=0$

$\underline{T}$

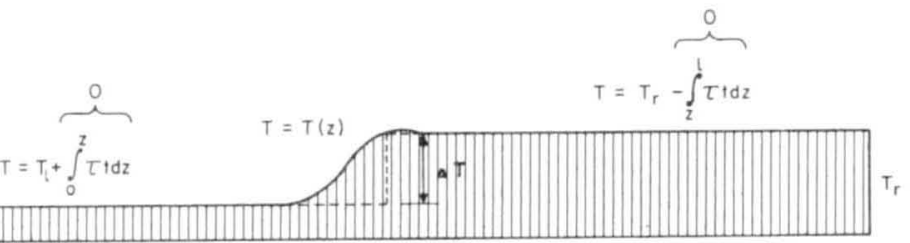

Bild 26 Verlauf der Spannungen und des Kantenschubes im Bereich des Querschnittssprunges eines Plattenbalkens unter reiner Biegung 
soll der Verlauf der Spannungen und des Kantenschubes im Bereich des Querschnittssprunges qualitativ skizziert werden. In Bild 26 ist ein Stück eines Plattenbalkens dargestellt, der durch zwei äussere Momente auf reine Biegung beansprucht ist, so dass keine Querkräfte vorhanden sind. Je nach Grösse des Querschnittssprunges ändem sich die Normalspannungen in einem Uebergangsbereich, der gleich gross sein kann wie die Querschnittshöhe.*) Auf beiden Seiten dieses Bereiches sind die Flanschkräfte verschieden gross und die Schubspannungen verschwinden im vorliegenden Fall. Durch die Aenderung der Normalspannungen treten in diesem Uebergangsbereich Schubspannungen auf. Das Integral der Schubkräfte $\tau$ tdz ist gleich der Aenderung der Flanschkräfte und somit der Kantenschubkräfte.

*) Mit Hilfe eines Programm über die Berechnung von Scheiben nach der Methode der "endlichen Elemente", welches von Herm Dr. K. KOVARI im Institut für Strassen- und Tunnel bau an der ETH entwickelt worden ist, wurde die Richtigkeit dieser Annahme bestätigt. 


\section{Berechnung des Beispiels in Bild 21}

Wegen der Symmetrie des Systems und der Belastung wird nur eine Hälfte betrachtet.
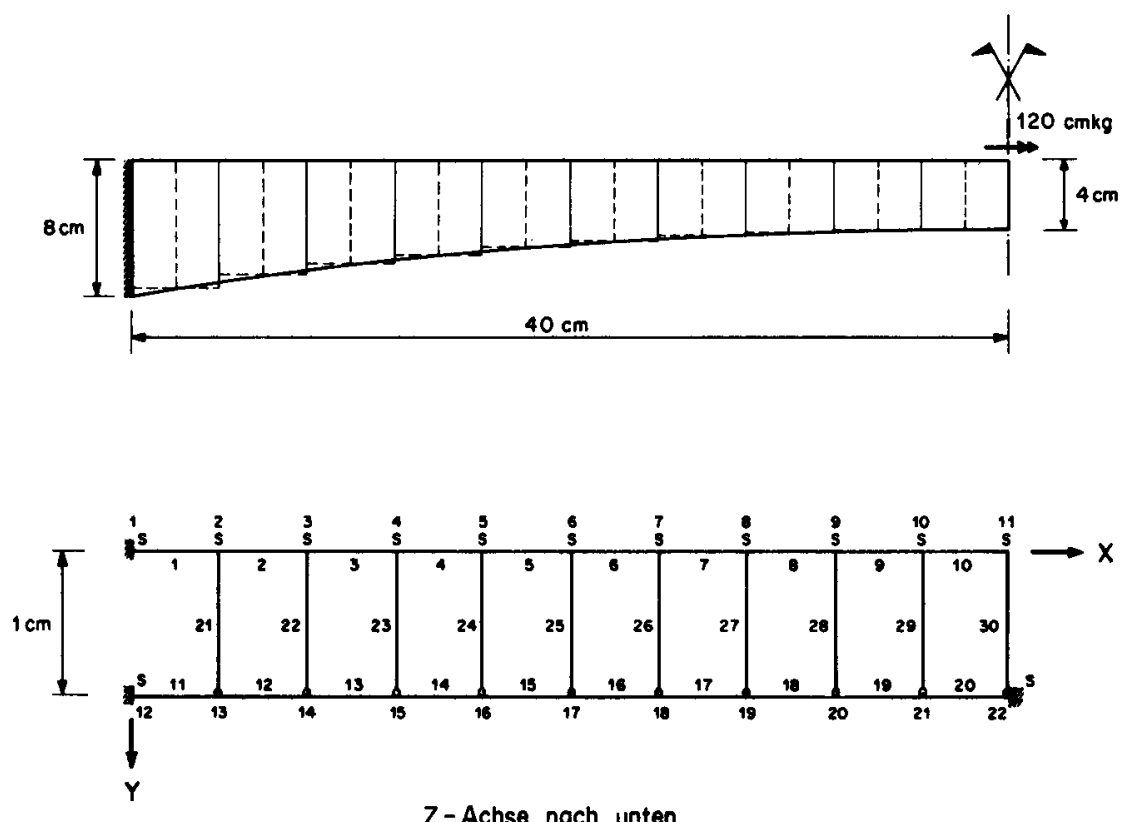

Z-Achse noch unten

Stabfestwerte fuir das STRESS - Programm:

$\begin{array}{clllll}\text { Stab } & \text { IX } & \text { IY } & \text { Stab } & \text { IX } & \text { IY } \\ 1 & 1.96 \mathrm{~cm}^{4} & \text { I000. } \mathrm{cm}^{6} & 11 & 10 . \mathrm{cm}^{4} & 2577.13 \mathrm{~cm}^{\circ} \\ 2 & 1.86 & 1000 . & 12 & 10 . & 1972.21 \\ 3 & 1.76 & 1000 . & 13 & 10 . & 1518.11 \\ 4 & 1.68 & 1000 . & 14 & 10 . & 1180.64 \\ 5 & 1.61 & 1000 . & 15 & 10 . & 932.79 \\ 6 & 1.56 & 1000 . & 16 & 10 . & 753.60 \\ 7 & 1.51 & 1000 . & 17 & 10 . & 627.13 \\ 8 & 1.48 & 1000 . & 18 & 10 . & 541.66 \\ 9 & 1.45 & 1000 . & 19 & 10 . & 488.97 \\ 10 & 1.44 & 1000 . & 20 & 10 . & 463.88\end{array}$

Stäbe 21 bis 30: IX $=100 \cdot \mathrm{cm}^{4} \quad I Y=100000 . \mathrm{cm}^{6}$ 
$\mathrm{E}=30000 . \mathrm{kg} / \mathrm{cm}^{2}, \quad G=10900 . \mathrm{kg} / \mathrm{cm}^{2}$ für alle Stäbe

Die äussere Belastung wird als Knotenlast in 2-Richtung im Punkt 22 angebracht: FORCE $z$ 60.

Die überzählige Grösse $X_{1}$ erzeugt im ganzen Träger Wölbmomente, die sich sprunghaft änderm. Diese treppenartige Wölbmomentenfläche wird im STRESS - Programm mit Knotenmomenten erzeugt.

\begin{tabular}{|c|c|c|c|}
\hline Stab & $-\mathbb{M}_{\omega}$ & Knoten & MOMENT Y \\
\hline 11 & $0 \quad \mathrm{~cm}^{2} \mathrm{~kg}$ & & \\
\hline 12 & 4.4 & 13 & 4.4 \\
\hline 13 & 8.4 & 14 & 4.0 \\
\hline 14 & 11.2 & 15 & 2.8 \\
\hline 15 & 13.6 & 16 & 2.4 \\
\hline 16 & 15.2 & 17 & 1.6 \\
\hline 17 & 18.0 & 18 & 2.8 \\
\hline 18 & 19.6 & 19 & 1.6 \\
\hline 19 & 20.2 & 20 & 0.6 \\
\hline 20 & 20.2 & 21 & 0. \\
\hline
\end{tabular}

Die Ordinaten der Momentenflächen (Bild 2l) lauten:

\begin{tabular}{|c|c|c|c|c|c|c|}
\hline \multirow[b]{2}{*}{ Knoten } & \multirow{2}{*}{$\frac{\text { Infolge }}{M_{\text {xo }}}$} & \multicolumn{2}{|c|}{$\mathrm{M}_{\mathrm{T}}=120 \mathrm{cmkg}$} & \multirow[b]{2}{*}{$\mathrm{M}_{\mathrm{x} 1}$} & \multicolumn{2}{|c|}{ Infolge $x_{1}=1 \mathrm{~kg}$} \\
\hline & & $\mathrm{T}_{\text {so }}$ & $\mathrm{M}_{\omega 0}$ & & $\mathrm{~T}_{\mathrm{s} 4}$ & $\mathrm{M}_{\omega 1}$ \\
\hline $\begin{array}{ll}1, & 12 \\
2, & 13 \\
3, & 14 \\
4, & 15 \\
5, & 16 \\
6, & 17 \\
7, & 18 \\
8, & 19 \\
9, & 20 \\
10, & 21 \\
11, & 22\end{array}$ & $\begin{array}{l}0 \\
0 \\
0 \\
0 \\
0 \\
0 \\
0 \\
0 \\
0 \\
0 \\
0\end{array}$ & $\begin{array}{l}0 \\
1.64 \\
3.02 \\
4.20 \\
5.26 \\
6.00 \\
6.35 \\
6.10 \\
5.21 \\
3.30 \\
0\end{array}$ & $\begin{array}{r}1437 \\
-1200 \\
-\quad 969 \\
-\quad 743 \\
-\quad 521 \\
-\quad 304 \\
-\quad 89 \\
126 \\
343 \\
566 \\
800\end{array}$ & $\begin{array}{l}-12 \\
-12 \\
-12 \\
-12 \\
-12 \\
=12 \\
=12 \\
-12 \\
=12 \\
-12 \\
-12\end{array}$ & $\begin{aligned} & 0 \\
- & 0.0165 \\
- & 0.0300 \\
= & 0.0405 \\
= & 0.0480 \\
- & 0.0520 \\
- & 0.0510 \\
- & 0.0445 \\
- & 0.0315 \\
- & 0.0160 \\
& 0\end{aligned}$ & $\begin{array}{r}16.75 \\
13.00 \\
9.00 \\
5.80 \\
3.40 \\
1.50 \\
-\quad 0.50 \\
-\quad 2.40 \\
-\quad 3.40 \\
-\quad 3.65 \\
-\quad 3.65\end{array}$ \\
\hline
\end{tabular}


Daraus werden die Formänderungsgrössen und die Ueberzählige bestimmt:

$$
x_{1}=-\frac{\delta_{10}}{\delta_{11}}=\frac{137,2}{19,8}=6,9 \mathrm{~kg}
$$




\begin{tabular}{|c|c|}
\hline d & DIstanz \\
\hline $\mathrm{h}$ & Scheibenhöhe \\
\hline$i, k$ & Laufindizes \\
\hline 1 & Stablänge \\
\hline $\mathrm{m}_{\mathrm{T}}$ & verteilt angreifendes Torsionsmoment \\
\hline $\mathrm{p}$ & verteilte Belastung \\
\hline $\mathrm{t}$ & Wandstärke \\
\hline $\mathrm{x}, \mathrm{y}$ & Koordinaten in der Querschnittsebene \\
\hline z & Koordinate in Richtung der Stabachse \\
\hline C & Schwerpunkt \\
\hline $\mathrm{D}$ & Schubmittelpunkt \\
\hline $\mathrm{E}$ & Elastizitätsmodul \\
\hline F & Querschnittsfläche \\
\hline G & Schubmodul \\
\hline$I_{x x}$ & Flächenträgheitsmoment \\
\hline$I_{\text {yy }}$ & Flächenträgheitsmoment $\quad=\int_{F} y^{2} d F$ \\
\hline$I_{\omega \omega}$ & sektorielles Trägheitsmoment $=\int \omega^{2} \mathrm{dF}$ \\
\hline $\mathrm{K}$ & Torsionskonstante \\
\hline $\mathrm{M}_{\mathrm{T}}$ & konzentriert angreifendes Torsionsmoment \\
\hline $\mathrm{M}_{\mathrm{X}}$ & Biegemoment $=\int_{F} \sigma x d F$ \\
\hline $\mathrm{M}_{\mathrm{y}}$ & Biegemoment $=\int_{F} \sigma_{\mathrm{y}} \mathrm{dF}$ \\
\hline $\mathrm{M}_{\omega}$ & Wölbmoment $=\iint_{F} \sigma \omega d F$ \\
\hline $\mathbb{N}$ & Normalkraft \\
\hline $\mathrm{P}$ & konzentrierte Belastung \\
\hline Q & Querkraft \\
\hline $\mathrm{T}$ & gesamtes Torsionsmoment \\
\hline $\mathrm{T}_{\mathrm{S}}$ & St. Venantsches Torsionsmoment \\
\hline $\mathrm{T}_{\boldsymbol{\omega}}$ & Wölbtorsionsmoment \\
\hline $\mathrm{T}_{\mathrm{k}}$ & Schubflussumme in der Kante $k$ \\
\hline & Widerstandsmoment \\
\hline
\end{tabular}




$\begin{array}{ll}\delta & \text { Formänderungsgrösse } \\ \zeta & \text { Verschiebung in z-Richtung } \\ \eta & \text { Verschiebung in y-Richtung } \\ \xi & \text { Verschiebung in x-Richtung } \\ \sigma & \text { Normalspannung } \\ \tau & \text { Schubspannung } \\ \varphi & \text { Stabverdrehung } \\ \omega & \text { Wölbkoordinate oder Einheitsverwölbung }\end{array}$


1 Wlassow, W.S.: "Dünnwandige elastische Stäbe". Bde 1 und 2. VEB Verlag für Bauwesen. Berlin 1964-65.

2 Kollbrunner, C.F., Basler, K.: "Torsion". Springer-Verlag, Berlin / Heidelberg/New York, 1966.

3 Cywinski, Z.: "Torsion des dünnwandigen Stabes mit veränderlichem, einfach symmetrischem, offenem Querschnitt". Der Stahlbau, Bd. 33, S. 301, 1964 und Bd. 36, S. 317, 1967.

4 Bazant, Z.P.: "Non-uniform Torsion of Thin-walled Bars of Variable Section". Abhandlung IVBH, Bd. 25, 1965.

5 Becker, G.: "Ein Beitrag zur statischen Berechnung beliebig gelagerter ebener gekrümmter Stäbe mit einfach symmetrischen dünnwandigen offenen Profilen von in Stabachse veränderlichem Querschnitt unter Berücksichtigung der Volbkrafttorsion". Der Stahlbau, Bd. 34, S. 334, 1965.

6 Lonkar, S.: "Bending and Torsion of Thin-walled Beams with Variable Open Cross-section". Diss. ETH 1968.

7 Lee, G.C., Szabo, B.A.: "Torsional Response of Tapered I-Girders". Proceedings of the American Society of Civil Engineers. Journal of the Structural Division, Vol. 93, No. ST 5, October 1967.

8 Bleich, F.: "Buckling Strength of Metal Structures". Mc Graw-Hill Book Company, New York / Toronto / Iondon, 1952 .

9 Bormscheuer, F.W.: "Beispiel und Formelsammlung zur Spannungsberechnung dünnwandiger Stäbe mit wölbbehindertem Querschnitt" . Der Stahlbau, Bd. 22, S. 32, 1953.

10 Anderheggen, E., Alberti, G., Iässker, A.: "Handbuch der Stress-Sprache". Institut für Baustatik ETH. Zürich, März 1967.

11 Thürlimann, B.: "Vorlesung Baustatik und Massivbau, Ausgewählte Kapitel" ETH, Zürich.

12 Schwyzer, H.: "Statische Untersuchung der aus ebenen Tragflächen zusammengesetzten räumlichen Tragwerke". Diss. ETH 1920.

13 Flügge, W.: "Handbook of Engineering Mechanics", Chapter 37 "Two-dimensional Problems" by Ch. Massonnet. 